\title{
A Survey on Beamforming Techniques for Wireless MIMO Relay Networks
}

\author{
Demosthenes Vouyioukas \\ Department of Information and Communication Systems Engineering, University of the Aegean, Samos, 83200 Karlovassi, Greece \\ Correspondence should be addressed to Demosthenes Vouyioukas; dvouyiou@aegean.gr
}

Received 24 July 2013; Accepted 3 October 2013

Academic Editor: Laurent Clavier

Copyright ( 2013 Demosthenes Vouyioukas. This is an open access article distributed under the Creative Commons Attribution License, which permits unrestricted use, distribution, and reproduction in any medium, provided the original work is properly cited.

\begin{abstract}
One of the major challenges the mobile broadband community faces is the exponential increase in mobile data traffic, even more so, for cell-edge users. Thus, in a multitier network, the demand for high-speed and interference-free transmission and reception is inevitable. Beamforming (BF) is an advanced technology that offers a significantly improved solution to reduce the interference levels and improve the system capacity. Accordingly, the establishment of relays in mobile data networks has emerged spectral efficiency enhancements and cell capacity gains from an overall system perspective. This paper provides a comprehensive survey focused on the performance of adopted beamforming technique on MIMO relay networks that is expected to overcome crucial obstacles in terms of capacity and interference. The main objective is to point out the state-of-the-art research activity on BF techniques in MIMO relay networks, under various network performance challenges. Thereby, it focuses on recently developed procedures for interference modeling and mitigation, BF channel modeling, channel estimation and feedback, complexity and power consumption, adaptive BF for multiuser relaying, degrees of freedom, diversity issues, and spectral efficiency, in cooperative and opportunistic systems. Different network topologies have been considered and categorized, pertaining the challenges of BF implementation in MIMO relay networks.
\end{abstract}

\section{Introduction}

Next-generation wireless networks are bound to offer a dramatic increase in data rate compared to the currently deployed networks. One major limiting factor towards this goal is the interference that arises due to the increased temporal and spectral reuse of resources. As a result, novel techniques that exploit the spatial domain will contribute significantly in the efficient operation of future networks. Among them, multiple-input multiple-output (MIMO) antenna configurations, cooperative relays, and beamforming (BF) have been very active research fields in the recent years, as they allow increased flexibility in interference mitigation.

Equipping transmitters and receivers with MIMO capabilities can achieve increased diversity and multiplexing gains. It was the seminal work of [1] which presented capacity results of MIMO systems in Gaussian channels that sparked great interest by the academia and the industry. The gains in capacity offered by MIMO topologies led to their inclusion in current and future wireless standards, namely, IEEE 802.11n, 802.16e, 3GPP LTE, and LTE-advanced. MIMO systems take advantage of the rich scattering observed in urban environments that offers independent propagation paths for the emitted signals. So, the designer of a MIMO system can target loading each antenna with a different information carrying signal, thus increasing the multiplexing gain or loading the same signal on all the antennas, thus improving the diversity gain. The capacity bounds of MIMO channels were the topic of [2] where realistic assumptions about time-varying channels and channel correlation were considered and results were given also for the multiple-access channel (MAC) and the broadcast channel (BC). The authors in [3] presented an overview of single-user (SU) MIMO and multiuser (MU) MIMO techniques and discussed the advantages offered by the latter at the cost of channel state information (CSI) at the transmitter in order to form accordingly the antenna beams. More recently, [4] provided an extension to multicell 
networks where MIMO nodes cooperate to exploit intercell interference. Cooperative techniques were introduced, such as base station (BS) cooperation and schemes that employed relays.

In general, the literature on cooperative relaying has seen a tremendous rise in contributions in recent years. Through cooperative relaying, coverage extension, increased reliability, and diversity can be harvested. The first study of the capacity of the relay channel was conducted in [5]. In addition, various relaying strategies such as amplifyand-forward (AF), decode-and-forward (DF), and compressand-forward (CF) were investigated in the seminal work of [6]. Optimization efforts for AF MIMO relay systems are depicted in [7], providing an overview of the fundamental results and practical implementation issues in designing $\mathrm{AF}$ MIMO relay systems. Relays are attractive as they improve three critical parameters of wireless networks. By allowing multihop transmission, transmitters are brought closer to the receiver, thus reducing the path loss attenuation of the signal. In addition, shadowing can be overcome by installing relay nodes in places where obstacles affect single-hop communications. Furthermore, multipath fading is mitigated through the provision of independent propagation paths. For example, even when one relay is employed, the signal propagates through a two-hop path and also through the direct path between the source and the destination. Also, cooperative relaying offers increased diversity [8], even when cooperative relays choose not to transmit but rather choose to cooperatively listen [9], thus improving the performance in terms of outage and error probability. Additionally, when multiple relays are available, selecting the best one according to instantaneous CSI was proven to be outage optimal in [10] compared to the case where multirelay transmissions are performed. More recently buffer-aided relays were examined [11] and relay selection schemes that aim at improved spectral efficiency were presented in $[12,13]$.

The third technique that is examined in this survey is beamforming. This technique uses $\mathrm{BF}$ matrices at the transmitters and the receivers, which form the antennas' beam patterns in such a way as to optimize a specific design criterion, such as mean square error (MSE) or signal-tonoise ratio (SNR). The implementation of $\mathrm{BF}$ requires the use of digital signal processors (DSPs) to shape accordingly the beam patterns that are emitted by the antennas. In the context of single-hop mobile communications, the article in [14] presented space-time processing to combat cochannel interference (CCI) and intersymbol interference (ISI). Another early work was [15], which proposed joint BF and power control in order to minimize the total transmitted power in the network while satisfying a signal-to-noiseplus-interference ratio (SINR) threshold at the receivers. An overview of smart antennas is given in [16] where switched beam, adaptive beam, and spatial division multiple-access (SDMA) are discussed. Moreover, digital signal processing algorithms such as direction-of-arrival (DoA) and adaptive $\mathrm{BF}$ were presented in [17].

This survey provides a detailed presentation of works that study BF techniques in networks where relays with MIMO capabilities are deployed. More specifically, the increased degrees of freedom (DoF) in exploiting the spatial resources are the main topic of the presented works. This area has seen a significant increase in contributions recently but a survey depicting its importance and categorizing these works has not been published except from a few articles, which provide quick overviews of some techniques. In [18], the authors present $\mathrm{BF}$ schemes for scenarios where MIMO AF relays assist the communication in single and multiuser networks either through one-way or two-way relaying. In another article [19], relay classification is performed and $\mathrm{BF}$ techniques are presented for various combinations of regenerative versus nonregenerative, full-duplex versus halfduplex, and one-way versus two-way. The author discusses the formulation of transmit-receive BF matrices, amplification matrices for nonregenerative relays, self-interference for full-duplex relays, and successive interference cancellation for the two-way case. Here, descriptions and categorizations for various network topologies and communication strategies are given. More specifically, articles that investigate BF schemes for single and multiuser communications are presented and their main contributions are highlighted. Also, a classification based on various relaying topologies such as single and multiple relaying as well as opportunistic relay selection is provided and the corresponding BF schemes are discussed. As the included works consider channel models and fading distributions that are examined in depth in the context of MIMO relaying, there is no channel modeling literature review in the context of this survey as emphasis is given on $\mathrm{BF}$ schemes and their various implementations.

This survey is organized by taking into consideration the different network topologies where $\mathrm{BF}$ techniques can be employed and its structure is as follows. In Section 2, the challenges of BF implementation in MIMO relay networks are given. More specifically, the design parameters that include MSE minimization and SNR maximization under various power constraints are presented. Also, overhead due to channel estimation and feedback is discussed. Another challenge that is often observed in MIMO networks is antenna correlation, thus its effect on the design of BF matrices is herein presented. In Section 3, BF schemes for singleuser communications are presented. Various different cases are presented, such as single and multiple relay topologies with and without relay selection. In Section 4, networks where multiuser communications take place are investigated and scenarios where single or multiple relays assist the communication for one or two-way communications are described. Discussion and open issues are the subject of Section 5, while conclusions are given in Section 6.

Notation. In this work, $(\cdot)^{T},(\cdot)^{H},(\cdot)^{*},|\cdot|,\|\cdot\|_{2}$, and $\|\cdot\|_{F}$ denote transpose, Hermitian transpose, complex conjugation, the determinant of a complex number, the Euclidean norm, and the Frobenius norm, respectively. Upper (lower) boldface letters will be used for matrices (vectors).

\section{Challenges}

To better illustrate the challenges that are presented in this section, the system model of [20] is adopted and extended 


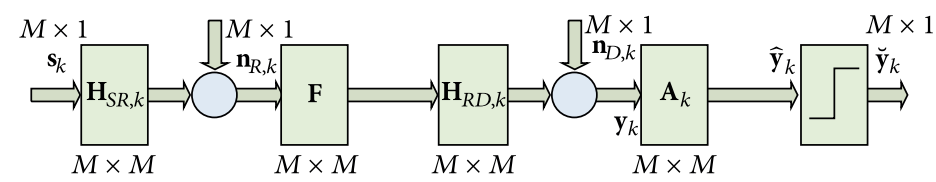

FIGURE 1: Block diagram of the two-hop relay equalization.

for two-hop topologies, as shown in Figure 1 [21], where a two-hop equalization process is depicted. The majority of works consider single-carrier transmission and reception but a multicarrier technology such as OFDM provides a more general system model. Furthermore, by focusing on a specific subcarrier, denoted by $k$, present works that use single-carrier block-fading channel models can be included. A network where a source communicates with a destination through an AF half-duplex relay is taken into consideration. However, the reader should note that adjustments should be made in the system model for the cases of DF and CF types of relaying, as there is no amplification in the analog domain by the relay. Furthermore, it is assumed that the source, the relay, and the destination have $M_{S}, M_{R}$, and $M_{D}$ antennas, respectively, and for simplicity $M_{S}=M_{R}=M_{D}=M$. The received signal at the destination is

$$
\begin{array}{r}
\mathbf{y}_{k}=\mathbf{H}_{R D, k} \mathbf{F H}_{S R, k} \mathbf{s}_{k}+\mathbf{H}_{R D, k} \mathbf{F} \mathbf{n}_{R, k}+\mathbf{n}_{D, k}, \\
1 \leq k \leq N,
\end{array}
$$

where $\mathbf{H}_{R D, k}$ is the $M \times M$ channel matrix of the RD link, $\mathbf{F}$ is the $M \times M$ amplification matrix at the relay, $\mathbf{s}_{k}$ is the $M \times 1$ transmitted signal vector from the source, $\mathbf{H}_{S R, k}$ is the $M \times M$ channel matrix of the SR link, and $\mathbf{n}_{R, k}, \mathbf{n}_{D, k}$ are $M \times 1$ additive white Gaussian noise (AWGN) vectors at the relay and the destination, respectively.

The transmitted signal vector from the source is defined as

$$
\mathbf{s}_{k}=\mathbf{B}_{k} \mathbf{x}_{k}=\sum_{i=1}^{L_{k}} \mathbf{b}_{k, i} \mathbf{x}_{k, i}
$$

where $\mathbf{x}_{k}$ are the $L_{k}$ transmitted symbols as $L_{k}$ established substreams are assumed and $\mathbf{B}_{k}$ is the $M \times L_{k}$ BF matrix at the source which can be designed jointly with the relay-amplify matrix $\mathbf{F}$ or separately and this holds also for the receiver's processing matrix $\mathbf{A}_{k}$. The transmitted symbols are assumed to satisfy the constraint $L_{k} \leq M$ as each node has $M$ antennas. At the destination, the received signal vector, assuming an equalizer, is expressed as

$$
\widehat{\mathbf{y}}_{k}=\mathbf{A}_{k}^{H} \mathbf{y}_{k} \text {. }
$$

In [21], equalization is performed first by designing the amplification matrix $\mathbf{F}$ at the relay and then for the overall channel through the MIMO equalizer $\mathbf{A}_{k}$. Another approach would consider joint optimization of $\mathbf{F}$ and $\mathbf{A}_{k}$. Various criteria can be considered in the optimization process and they are discussed as follows.
2.1. Performance Criteria. In the works that investigate the formulation of BF matrices, various performance criteria have been proposed. More specifically, transmit and receive $\mathrm{BF}$ matrices are derived through optimization problems that are subject to various performance criteria.

(a) Minimization of the Mean Square Error (MMSE). Many works take into consideration this important metric, which aims at the minimization of the estimation errors at the destination under a target SNR. Articles presenting problems with MMSE are included in [21-30]. In [20], a multicarrier system has been presented and the MSE for the $k$ th carrier is defined as the trace of the covariance matrix of the error vector $\mathbf{e}_{k} \triangleq\left(\widehat{\mathbf{x}}_{k}-\mathbf{x}_{k}\right)$, where $\widehat{\mathbf{x}}_{k}$ is the estimation of $\mathbf{x}_{k}$. As a result, the problem of MSE minimization requires BF matrices to provide

$$
\min \mathbf{E}_{k}\left(\mathbf{B}_{k}, \mathbf{F}, \mathbf{A}_{k}\right) \triangleq \min \mathbf{E}\left[\left(\widehat{\mathbf{x}}_{k}-\mathbf{x}_{k}\right)\left(\widehat{\mathbf{x}}_{k}-\mathbf{x}_{k}\right)^{H}\right] .
$$

The MSE of the substreams are the diagonal elements of $\mathbf{E}_{k}$, so for the ( $k$ th, $i$ th) substream, its MSE value will be located at the ith diagonal position. As a result, MSE values can be denoted through the trace of $\mathbf{E}_{k}$.

(b) Minimization of the Sum of MSE. In [31, 32], two end nodes communicate simultaneously through a MIMO AF relay. So, the minimization of MSE at both directions is considered; thus the optimization target is transformed into the minimization of the sum of MSE as follows:

$$
\begin{aligned}
\min & \mathbf{E}_{1 k}\left(\mathbf{B}_{1 k}, \mathbf{B}_{2 k}, \mathbf{F}, \mathbf{A}_{1 k}, \mathbf{A}_{2 k}\right) \\
& +\min \mathbf{E}_{2 k}\left(\mathbf{B}_{1 k}, \mathbf{B}_{2 k}, \mathbf{F}, \mathbf{A}_{1 k}, \mathbf{A}_{2 k}\right) \\
& \triangleq \min \mathbf{E}\left[\left(\widehat{\mathbf{x}}_{1 k}-\mathbf{x}_{2 k}\right)\left(\widehat{\mathbf{x}}_{1 k}-\mathbf{x}_{2 k}\right)^{H}\right] \\
& +\min \mathbf{E}\left[\left(\widehat{\mathbf{x}}_{2 k}-\mathbf{x}_{1 k}\right)\left(\widehat{\mathbf{x}}_{2 k}-\mathbf{x}_{1 k}\right)^{H}\right],
\end{aligned}
$$

where indices 1 and 2 are used to denote matrices and signals which correspond to the two end nodes which are concurrently communicating through the relay.

(c) Maximization of SINR. Other works form optimization problems by considering the maximization of the SNR or SINR in the cases of multiuser and two-way relaying networks. From [20], the SINR for the $i$ th spatial substream of the $k$ th subcarrier, ( $k$ th, $i$ th) substream, is given in relation to MSE as

$$
\operatorname{SINR}_{k, i}=\frac{1}{\mathrm{MSE}_{k, i}}-1,
$$


where $\mathrm{MSE}_{k, i}$ is the MSE of the ( $k$ th, $i$ th) substream and corresponds to the $i$ th diagonal element of $\mathbf{E}_{k}$.

It is obvious that the maximization of the SINR is equivalent to the minimization of the MSE. The articles which optimize the BF matrices under the maximization of SNR are [21, 33-39].

When interference arises, SNR is replaced by SINR by considering streams that interfere in the reception of the desired signal. Many works consider zero-forcing (ZF) as in $[28,40-43]$. The goal of $\mathrm{ZF}$ is to cancel the interference at the receiver and is based on the ZF filter, which uses the pseudoinverse matrix of the channel matrix between the communicating nodes as follows:

$$
\mathbf{Z}=\mathbf{H}^{+}=\left(\mathbf{H}^{H} \mathbf{H}\right)^{-1} \mathbf{H}^{H}
$$

(d) Maximization of Capacity. Various articles target capacity maximization, as is the case in [21,27,44-57]. The relation of the maximization of mutual information to MSE is given as [20]

$$
I=-\log |\mathbf{E}| .
$$

From this equation, it is concluded that the maximization of the mutual information derives from the minimization of the MSE.

(e) Maximization of the Distance of Network-Coded Symbols. Other works employ network coding (NC) and consider the maximization of the minimum symbol distance as a design criterion. In two-way relay networks, uncoded symbols are transmitted simultaneously by the two end nodes and received by the relay. Next, the relay broadcasts an $\mathrm{NC}$ version of the two symbols, such as an XOR combination, in order for the end nodes to decode their signal of interest. The minimum distance of the different network-coded symbols is given as [58]

$$
d_{\min }^{(\mathrm{NC})}=\min \left(a_{1}, a_{2}\right) \cdot d_{\min },
$$

where $a_{1}, a_{2}$ are the $\mathrm{BF}$ gains of each end node, chosen to align the signal subspaces at the relay and $d_{\text {min }}$ is the minimum distance of the transmit constellation $S$, which contains MQAM symbols. These works include [58, 59].

2.2. Power Constraints. A critical parameter in designing BF schemes is the power constraint imposed on different nodes in the system. Power constraints are practical considerations as network nodes may be battery-operated and regulatory authorities define maximum power levels for transmission in wireless systems. The following focus on the $k$ th subcarrier as most works consider single-carrier transmissions in their system models.

(a) Power Constraint at the Relay(s). Many works impose various types of power constraints at the relays depending on their number and whether or not multi- or single-relay transmissions take place. For single-relay topologies, [23, 27,
$28,40,45,51,52]$ impose a power constraint at the single relay expressed by

$$
\left\|\mathbf{F H}_{S R, k} \mathbf{B}_{k}\right\|_{2}^{2}+\sigma_{R, k}^{2}\|\mathbf{F}\|_{F}^{2} \leq P_{2, k} .
$$

When multiple relays are used, individual and sum-power constraints are imposed. The algorithms in $[33,42,60]$ impose an individual power constraint of the form

$$
\left\|\mathbf{F H}_{S R_{j}, k} \mathbf{B}_{k}\right\|_{2}^{2}+\sigma_{R_{j}, k}^{2}\|\mathbf{F}\|_{F}^{2} \leq P_{2, R_{j}, k}
$$

at each relay $j$, while $[21,22,43,44,46,56,61]$ employ sumpower constraints of the form

$$
\sum_{j=1}^{N_{R}}\left\|\mathbf{F H}_{S R_{j}, k} \mathbf{B}_{k}\right\|_{2}^{2}+\sigma_{R_{j}, k}^{2}\|\mathbf{F}\|_{F}^{2} \leq P_{2, k}
$$

Finally when one relay is selected from a set of available relays, [62] sets a power constraint at the selected relay, that is, similar to the constraint of the single relay topologies.

(b) Power Constraint at the Source and the Relays. Other works search for BF matrices under power constraints at both the source(s) and the relay(s). In networks where a single relay is available, [24-26, 34, 48, 49] have individual power constraints for the source and the relay, and the source's constraint is defined as $\left\|\mathbf{B}_{k}\right\|_{2}^{2} \leq P_{1}$. In a multiple-relay scenario, [61] considers individual constraints for the source and the relays that operate under a sum-power constraint. Also, for similar use, cases [33, 38, 39] impose joint power constraints at the source and the relays expressed by

$$
\left\|\mathbf{B}_{k}\right\|_{2}^{2}+\sum_{j=1}^{N_{R}}\left\|\mathbf{F H}_{S R_{j}, k} \mathbf{B}_{k}\right\|_{2}^{2}+\sigma_{R_{j}, k}^{2}\|\mathbf{F}\|_{F}^{2} \leq P_{k} .
$$

In topologies where relay selection is performed, [36] imposes a separate power constraint at the source and the selected relay while [47] has individual power constraints at the source and the selected set of relays. For multiplesource scenarios where a single relay is available, the works in $[29,31,53-55,58,59]$ consider individual and separate power constraints at the sources and the relay. In networks where multiple relays are employed, [41, 63, 64] have individual power constraints at the sources and a sum power constraint at the relays,while [50] imposes individual sum-power constraints at the sources and the relays.

(c) Power Constraint at the Receiver Side. In this category, for multiple source-destination pairs, the target is the minimization of the interference which is received at each destination as in $[21,42]$. For a similar setup, the maximization of the desired signal power is the goal of [57].

2.3. Complexity. Many works present optimal BF schemes; however, in practical setups, these techniques are very difficult to implement due to the induced computational complexity. As a result, there have been a number of suboptimal methods that do not significantly degrade the network's 
performance. For the optimization of the BF vectors, there are several published papers, providing reduced complexity and thus enhancing some of the aforementioned performance criteria.

For single-user communications, various works provide efficient algorithms for the calculation of the BF matrices. In $[33,61]$, the authors provide two suboptimal methods which optimize the source BF vectors and can be used in networks with larger scale than the three-node networks under study. The first suboptimal solution is based on the gradient method that finds the local optimum, and the second uses maxmin optimization that leads to a semidefinite programming problem whose solution can be obtained. Furthermore, the authors of [22] provide two suboptimal techniques, which offer simpler solutions to the power allocation problem at the relays. In the first, equal power allocation to all the frequencies is employed while the second performs equal power allocation to all the frequencies and the relays, thus significantly reducing the amount of channel estimation overhead. Also, in [24], the optimization problem that is formed by the transceiver design is nonconvex and the authors provide a decomposition method that transforms the optimization problem into a master problem and a subproblem. The master problem is formulated as a relayprecoder design problem, whereas the subproblem is a source-precoder design problem. By solving the subproblem, the source precoder is obtained and then the solution is transferred to the master problem for the derivation of the relay precoder.

Other works provide reduced complexity BF algorithms for multiuser communication networks. In [38, 39], the system performance is improved through a low-complexity $\mathrm{BF}$ vector optimization technique that targets the maximization of the effective channel gains. By considering the relaying functionality of the two destinations as an auxiliary mechanism, the low-complexity algorithm focuses on the maximization of the broadcast channel gains. This is achieved by initializing the combining vectors according to a blind algorithm and then by updating them as the eigenvectors corresponding to the largest eigenvalue for the two SD channels. Moreover, in [48], a MIMO broadcast relay channel is studied targeting sum-rate maximization. It is shown that the problem of finding the input covariance matrices and the relay $\mathrm{BF}$ matrix is nonconvex and in order to solve it the authors propose to examine the dual multiple access relay channel (MARC). By matching the relay BF matrix to the left and right singular vectors of the first and second hop channels, the solution to this problem is tractable and sumrate optimization is performed for this case. The MIMO broadcast relay channel is also the topic of [49] and the goal is to maximize the weighted sum rate. However, finding the source precoding matrix $\mathbf{B}$ and the relay $\mathrm{BF}$ matrix $\mathbf{F}$ is a non-linear and nonconvex problem. To achieve a tractable solution, the authors set an equivalent problem that aims to minimize MSE. This problem consists of four variables, $\mathbf{B}, \mathbf{F}$, the receive matrix $\mathbf{A}_{k}$ of user $k$, and the weight matrix $\mathbf{W}_{k}$ of user $k$. By keeping three of the four variables fixed, the problem is convex with respect to the remaining variable and has a closed-form solution. Another work [50] examines the relay interference broadcast channel and targets the end-to-end rate maximization. The proposed low-complexity algorithm is performed through a three-step procedure. During the first step, the precoders at the relays are designed in order to maximize the second-hop sum rates. In the second step, using the knowledge of the second-hop rates and the time-sharing value, that is, the fraction of time where each hop is performed, the source precoders are designed and an approximation of the optimal end-to-end rate is achieved. In the last step, power control is employed to balance any rate mismatch. The uplink case of a cellular network is studied in [26] where multiple users communicate with one BS through one RS. This algorithm is proposed when the number of the transmitted independent streams from the users is greater than or equal to their number of antennas, that is, for fully loaded scenarios.

For networks where multiple source-relay-destination links are present, various less complex algorithms have been proposed. In [56], the authors study a two-hop topology with multiple MIMO relays. As the optimal sum-rate maximization BF strategy introduces increased complexity, a reduced complexity suboptimal scheme is presented. This iterative algorithm decouples the effective channels and aligns their channel gains at the same level, thus offering a tractable solution to the sum-rate maximization problem. Moreover, another scheme based on interference neutralization is given which cancels the interference at the last hop. Moreover, in [57], the authors present an approximation in the computation of the end-to-end rate in a multisource multidestination network with relays. Through this approximation, the relationship between the two-hop channel gains are taken into account and suboptimal solutions can be achieved when designing the relay BF matrix. In this way, rate mismatch is avoided as the dominance of a specific two-hop is considered and the end-to-end sum rates are improved.

When two or more end nodes communicate with each other simultaneously, two-way communication occurs and many other works present suboptimal algorithms in such cases. In [58], the authors study a two-way MIMO relay network that employs network coding. More specifically, they aim to improve the network's performance by maximizing the minimum distance of the NC symbols. As the derivation of the global optimum depends on the individual constellation and their mapping rule, a closed-form solution for the transmit precoders at the end nodes is not known. In order to reduce the complexity of optimization, a suboptimal precoding strategy that consists of elements of three different precoders is proposed. The resulting precoding strategy adapts between precoding with subspace alignment, precoding with subspace separation, and precoding with maximum ratio transmission. In addition, [29] considers linear MMSE receivers in a two-way MIMO relay network. As the joint source, relay, and receiver matrices optimization depends on multiple matrix variables, a suboptimal relay precoding matrix design is presented. The suboptimal algorithm is proposed in cases where the relay has more or equal number of antennas than both end nodes. To reach to a tractable solution, the main problem is decomposed into subproblems, which are solved using the projected gradient algorithm. 
Furthermore, in [31], a simplified algorithm is proposed which computes transmit and receive BF matrices at the end nodes of a two-way network. This is achieved by computing the receive $\mathrm{BF}$ matrices given all other $\mathrm{BF}$ vectors and then computing the transmit BF matrices through one-step SVD decomposition. Finally, the relay BF matrix is obtained. The authors of [53] give a suboptimal solution to the sum-rate optimization problem in a two-way MIMO relay network. As the original problem is nononvex, a decomposition into three separate subproblems is proposed to find the transmit, receive, and relay $\mathrm{BF}$ matrices. However, the problem of finding the relay $\mathrm{BF}$ matrix is nonconvex and an approximation is given based on the power iteration technique. Also, [54] examines a two-way MIMO relay network where suboptimal schemes to compute the relay BF matrix structure are presented. The first is based on the combination of maximal ratio reception and maximal ratio transmission while the second on ZF reception and ZF transmission. Finally, in [27], a multiuser two-way relay network is examined where precoding design aims to suppress co-channel interference. In contrast to BS precoding design with a fixed RS precoder, the design of the RS precoder with a fixed BS precoder is nonconvex. As a result, the authors propose an iterative algorithm that finds a local optimal solution either through eigenvalue decomposition or through randomization, which leads, in a quasioptimal solution.

All the aforementioned papers are categorized accordingly in Table 1 with respect to their network configuration, depicting the complexity in quantitative way (whenever possible) and elsewhere in qualitative way, and the utilized suboptimal optimization method, along with the detailed configuration and the relay strategy scheme that they adopt.

2.4. Channel Estimation and Feedback. The formulation of transmit and receive BF matrices is based on successful channel estimation and the feedback of CSI to the nodes that perform BF. The works presented in this subsection consider cases where full CSI is not available and, so, efficient methods that are based on partial CSI are devised to allow BF techniques to take place. Furthermore, the operation of BF with reduced CSI exchange minimizes additional overhead to the network and allows increased QoS.

In scenarios where only a single destination is present, the following works aim to give BF algorithms with reduced CSI exchange, in order to provide scalable methods for more complex network topologies. In [65], clusters of multiple-antenna relays assist the communication of a multiple-antenna source and a single-antenna destination. In addition, transmit maximal ratio combining $\mathrm{BF}$ (TMRC-BF) is employed at the relays which double the duration of the effective channel through which the transmitted signal propagates. As a result, additional overhead is needed due to pilot signals that are used to estimate the channel. By using the real value property of the equivalent channel, the pairing of relay clusters is proposed in conjunction with corresponding pilot designs. Simulations for MSE performance reveal that when the pilot SNR is not less than $5 \mathrm{~dB}$ below the pilot SNR during TMRC$\mathrm{BF}$, optimal performance can be achieved. In [34], a modified quantization scheme at the destination is presented in order to reduce the amount of CSI feedback. This scheme is based on the fact than in the extreme cases where the SD link or the SRD link is weak, no feedback or only the knowledge of the right singular vector of the direct link is required at the relay to determine the source BF vector. The article in [66] proposes antenna selection in order to reduce the overhead of feedback compared to multiple-antenna BF. The authors map this scenario to a case where a dominant channel is present or when diversity is preferred compared to multiplexing gain. The antenna selection with limited feedback is described where narrowband tones are transmitted from each source and relay antennas and at the end the destination is able to perform MMSE. This selection process requires $\log _{2}\left(M_{S} M_{R}\right)$ bits of feedback, where $M_{S}$ and $M_{R}$ are the number of antennas at the source and relay, respectively, and $M_{R}+2 M_{S}$ are time slots for SNR estimation; thus the minimization of SNR estimation time is important in this scheme. From the analysis, it is shown that full diversity order can be achieved.

When relay selection takes place, instantaneous channelgain values are needed and CSI availability is critical for the performance of the selection algorithm. In [35], the authors consider the relay selection scheme as a special case of BF where limited feedback equal to $\log _{2}(m)$, where $m$ is the number of relays, is needed. The compared schemes include relay selection and multiple-relay $\mathrm{BF}$ with various amounts of feedback ranging. From the results, it is concluded that the selection scheme achieves better performance than BF for limited feedback cases in AF relay networks. Also, in [36], partial relay selection (PRS) is employed in order to reduce the CSI requirements of the optimal opportunistic relay (OR) selection. More specifically, the selection process is based on the quality of the SR links. In this way, no additional CSI feedback from the RD links is required. Comparisons with OR with full CSI indicate that when $\min \left(M_{r, 1} M_{d}, M_{r, 2} M_{d}\right) \geq$ $M_{s} M_{r, 1}+M_{s} M_{r, 2}$, where $M_{s}, M_{d}$ and $M_{r, q}$ are, respectively, the numbers of antennas at the source, destination, and the $q$ th relay, PRS and OR achieve the same diversity gain.

For a network where one BS serves multiple users, the authors in [67] study user selection based only on partial CSI of transmit correlation. As a result, in each transmission, one user is served by the BS and one by the RS, and their selection is based on an orthogonal pair of users which has the largest phase difference of the transmit correlation, in order to reduce the interference received by each user and maximize the achievable sum rate of multiuser dual-hop MISO relay channels.

2.5. Antenna Correlation. Another challenge for MIMO BF is the correlation between the antennas at each node. Such scenarios are of great importance as colocated antennas in small devices such as smartphones result in spatial correlation in transmission and reception.

In [68], a network with a MIMO source, a single-antenna fixed gain relay, and a MIMO destination is explored. The analysis takes into account the correlation between the antennas at the source and at the destination using the Kronecker correlation model. Closed-form expressions are derived for 
TABLE 1: Classification of articles based on network topology, with their corresponding complexity and suboptimal optimization method used.

\begin{tabular}{|c|c|c|c|}
\hline $\begin{array}{l}\text { Reference } \\
\text { article }\end{array}$ & Complexity & Suboptimal optimization method & $\begin{array}{l}\text { Configuration/relay } \\
\text { strategy }\end{array}$ \\
\hline \multicolumn{4}{|c|}{ Single relay/single user } \\
\hline$[24]$ & $\begin{array}{l}\text { The cost function that expresses the } \\
\text { minimization of MSE is a nonlinear } \\
\text { function of the two precoding matrices at } \\
\text { the source and the relay, resulting in a } \\
\text { nonconvex optimization problem }\end{array}$ & $\begin{array}{l}\text { Source-precoder subproblem is solved by } \\
\text { applying Karush-Kuhn-Tucker (KKT) } \\
\text { conditions to single relay-precoder } \\
\text { optimization }\end{array}$ & $\begin{array}{l}\text { S: single MIMO } \\
\text { R: single AF MIMO } \\
\text { D: single MIMO }\end{array}$ \\
\hline$[54]$ & $\begin{array}{l}\text { MRR-MRT: proportional to MF-based } \\
\text { receive and transmit beamforming to } \\
\text { maximize the total signal power } \\
\text { ZFR-ZFT: proportional to ZF-based } \\
\text { receive and transmit beamforming to } \\
\text { remove the interference }\end{array}$ & $\begin{array}{l}\text { Relay BF matrix calculations based on: } \\
\text { (i) Maximal-Ratio Reception and } \\
\text { Maximal-Ratio Transmission } \\
\text { (MRR-MRT) } \\
\text { (ii) Zero-Forcing Reception and } \\
\text { Zero-Forcing Transmission (ZFR-ZFT) }\end{array}$ & $\begin{array}{l}\text { S: single-antenna, single } \\
\text { source } \\
\text { R: single AF two-way } \\
\text { single-pair MIMO } \\
\text { D: single-antenna, single } \\
\text { user }\end{array}$ \\
\hline [31] & $\begin{array}{l}O\left(N^{3}\right), N \text { : number of antennas at the } \\
\text { source }\end{array}$ & $\begin{array}{l}\text { Optimal beamforming at all nodes based } \\
\text { on the minimization of the sum MSE } \\
\text { adopting KKT conditions }\end{array}$ & $\begin{array}{l}\text { S: single MIMO } \\
\text { R: single AF two-way } \\
\text { single-pair MIMO } \\
\text { D: Single MIMO }\end{array}$ \\
\hline [53] & $\begin{array}{l}\text { The sum-rate maximization problem in } \\
\text { this two-way AF single-relay network is } \\
\text { not convex and an approximate solution } \\
\text { can be derived through decomposition }\end{array}$ & $\begin{array}{l}\text { Joint optimization of the transceivers at } \\
\text { both sources and relay in terms of } \\
\text { sum-rate maximization and based on } \\
\text { KKT conditions }\end{array}$ & $\begin{array}{l}\text { S: single MIMO } \\
\text { R: single AF two-way } \\
\text { single-pair MIMO } \\
\text { D: single MIMO }\end{array}$ \\
\hline [58] & $\begin{array}{l}\text { The global optimum regarding the } \\
\text { maximization of the distance of } \\
\text { network-coded symbols is complicated to } \\
\text { be found, as it depends on the symbol } \\
\text { constellation and the corresponding } \\
\text { mapping rule. Moreover, for general } \\
\text { MIMO channels between the two sources } \\
\text { and the relay, a closed-form solution has } \\
\text { not been derived }\end{array}$ & $\begin{array}{l}\text { Design of a hybrid precoder combining } \\
\text { three different classes of suboptimal } \\
\text { precoders, with additional constraints of } \\
\text { subspace alignment, subspace separation, } \\
\text { and the maximal ratio transmission } \\
\text { Define the optimal precoding vectors } \\
\text { within each class in terms of maximizing } \\
\text { the minimum distance between different } \\
\text { network coding symbols }\end{array}$ & $\begin{array}{l}\text { S: single MIMO } \\
\text { R: single AF two-way } \\
\text { single-pair MIMO } \\
\text { D: Single MIMO }\end{array}$ \\
\hline
\end{tabular}
Multiple relays/single user

(i) frequency domain $(\mathrm{FD})$ based processing at the relays

For each relay: $\log _{2}\left(N_{c}\right) N N_{c} / 2+N^{2} N_{c}$ $N_{c}$ : iid symbols

$N$ : number of antennas at the relay

[61]
The optimization of the source BF matrix is nonconvex

The joint source, relay and receive matrices optimization problem that aims at two-way MSE minimization is non-convex. The global optimum cannot be achieved with reasonable complexity (nonexhaustive searching)

Depending on the imposed power constraints, the optimization problems for each optimal case induce different complexity. When multiple relays are employed, the optimization is nonconvex for the case of joint relay power constraints and joint source-relay power constraints (ii) equal power allocation (EPA) across all frequencies

(iii) equal power allocation (EPA) across all frequencies and relays

Gradient algorithm for finding local optimum of the source BF vector

Iterative algorithm for joint source, relay, and receive matrices optimization for two-way sum MSE minimization

Max-min optimization of the source BF vector under joint relay and jointed source-relay power constraints:

(i) transformation method

(ii) gradient method

(iii) relaxation method
S: single-antenna, single source

R: multiple AF MIMO

D: single-antenna, single user

S: single MIMO

R: Multiple AF MIMO

D: single-antenna, single user

S: single MIMO

R: multiple AF two-way single-pair MIMO

D: single MIMO

S: single MIMO

R: multiple AF MIMO

D: single-antenna, single user 
TABle 1: Continued.

\begin{tabular}{|c|c|c|c|}
\hline $\begin{array}{l}\text { Reference } \\
\text { article }\end{array}$ & Complexity & Suboptimal optimization method & $\begin{array}{l}\text { Configuration/relay } \\
\text { strategy }\end{array}$ \\
\hline \multicolumn{4}{|c|}{ Single relay/multiple users } \\
\hline [26] & $\begin{array}{l}\text { Proportional to the beamforming } \\
\text { algorithm for the fully loaded or } \\
\text { overloaded uplink }\end{array}$ & $\begin{array}{l}\text { Linear MMSE criterion for both } \\
\text { downlink/uplink utilizing iterative } \\
\text { beamforming algorithm: } \\
\text { (i) equalizer design at the user/BS } \\
\text { (ii) forwarding matrix design at the relay } \\
\text { station } \\
\text { (iii) precoder design at the BS/user }\end{array}$ & $\begin{array}{l}\text { S: single MIMO } \\
\text { R: single AF MIMO } \\
\text { D: multiple MIMO users }\end{array}$ \\
\hline$[38,39]$ & $\begin{array}{l}2^{2 B} \text { for each channel matrix (SR, RD, RR) } \\
B \text { : bits }\end{array}$ & $\begin{array}{l}\text { (i) blind algorithm } \\
\text { (ii) broadcast channel optimization }\end{array}$ & $\begin{array}{l}\text { S: single MIMO } \\
\text { R: single AF MIMO } \\
\text { D: multiple MIMO users }\end{array}$ \\
\hline [48] & $\begin{array}{l}\text { The formulated sum-rate optimization is } \\
\text { non-convex and a global optimal solution } \\
\text { cannot be obtained }\end{array}$ & $\begin{array}{l}\text { Optimization of the dual multiple access } \\
\text { relay channel (MARC) applying } \\
\text { alternating minimization algorithm } \\
\text { (AMA) that maximizes the network sum } \\
\text { rate }\end{array}$ & $\begin{array}{l}\text { S: single MIMO } \\
\text { R: single AF MIMO } \\
\text { D: multiple MIMO users }\end{array}$ \\
\hline [49] & $\begin{array}{l}\text { Proportional to two linear relay } \\
\text { beamforming schemes }\end{array}$ & $\begin{array}{l}\text { Weighted MMSE method for MSE } \\
\text { minimization }\end{array}$ & $\begin{array}{l}\text { S: single MIMO } \\
\text { R: single AF MIMO } \\
\text { D: single-antenna, Multiple } \\
\text { users }\end{array}$ \\
\hline [27] & $\begin{array}{l}n_{\mathrm{BS}}=(N K+1)^{2}(K+2)^{0.5}\left(2 N K+K^{2}+\right. \\
2 K+4) \log (1 / \varepsilon) \\
n_{\mathrm{RS}}= \\
l_{\mathrm{RS}}\left(\max \left(M^{2}, K+2\right)^{4} M \log (1 / \varepsilon)+n_{\mathrm{rd}}\right) \\
N: \text { number of BS antenna } \\
M: \text { number of relay antenna } \\
K: \text { number of MS single antenna } \\
l_{\mathrm{RS}}: \text { iteration number in Algorithms } 1 \text { and } \\
2 \\
\end{array}$ & $\begin{array}{l}\text { (i) Iterative algorithm for RS precoding } \\
\text { design with the BS precoder fixed } \\
\text { (ii) Design of joint BS-RS precoding by } \\
\text { solving the BS and RS precoding } \\
\text { alternately }\end{array}$ & $\begin{array}{l}\text { S: single MIMO } \\
\text { R: single AF two-way } \\
\text { multi-pair MIMO } \\
\text { D: single-antenna, multiple } \\
\text { users }\end{array}$ \\
\hline
\end{tabular}

Multiple relays/multiple users

(i) The complexity of the centralized adaptive $\mathrm{BF}$ is $O\left(J\left(\sum_{k} m_{k}^{2}\right)^{2}\right)$ per iteration

$J$ : is the number of sources and destination nodes

$m_{k}$ : is the number of antennas at the $k \mathrm{th}$ relay

(ii) For the decentralized algorithm, the complexity per iteration is equal to $O\left(J m_{i}^{4}\right)$ at the $i$ th relay

[43] (i) Centralized adaptive BF algorithm with the existence of a local processing center connected to all the relays and minimizing a cost function using state-space modeling approach (ii) Decentralized adaptive BF algorithm allowing each relay terminal to compute its beamforming matrix locally with limited amount of data exchange with the other relays, employing Kalman filtering to estimate its beamforming coefficients iteratively

$\mathrm{ZF}-\mathrm{BF}$ is used in order to reduce complexity by projecting the $\mathrm{BF}$ vector to a low dimensional space thus reducing the number of variables that are used for optimization

(i) Sum-rate maximization through an iterative algorithm subject to a sum-power constraint of the relay BF matrices

(ii) Interference neutralization beamforming scheme subject to a linear constraint on the desired signals
S: single-antenna, Multiple sources

R: multiple AF MIMO

D: single-antenna, Multiple users

S: single-antenna, multiple sources

R: multiple AF MIMO

D: single-antenna, multiple users

S: single-antenna, multiple sources

R: Multiple AF MIMO

D: single-antenna, multiple users 
TABle 1: Continued.

\begin{tabular}{|c|c|c|c|}
\hline $\begin{array}{l}\text { Reference } \\
\text { article }\end{array}$ & Complexity & Suboptimal optimization method & $\begin{array}{l}\text { Configuration/relay } \\
\text { strategy }\end{array}$ \\
\hline$[50]$ & $\begin{array}{l}\text { Proportional to three-phase cooperative } \\
\text { algorithms with distributed } \\
\text { implementation }\end{array}$ & $\begin{array}{l}\text { Sum-utility maximization via } \\
\text { matrix-weighted sum-MSE Minimization } \\
\text { for end-to-end sum-rate maximization }\end{array}$ & $\begin{array}{l}\text { S: multiple MIMO } \\
\text { R: multiple AF MIMO } \\
\text { D: multiple MIMO users }\end{array}$ \\
\hline [57] & $\begin{array}{l}\text { The sum-rate optimization problem is } \\
\text { NP-hard and the global optimal solution } \\
\text { cannot be derived with realistic } \\
\text { computation complexity }\end{array}$ & $\begin{array}{l}\text { Distributed two-hop interference pricing } \\
\text { algorithm for relay beamforming design } \\
\text { for maximizing end-to-end sum rates }\end{array}$ & $\begin{array}{l}\text { S: multiple MIMO } \\
\text { R: multiple AF MIMO } \\
\text { D: multiple MIMO users }\end{array}$ \\
\hline
\end{tabular}

S: source, R: relay, D: destination.

the outage probability and BER performance. Moreover, the diversity order of the network is found to be dependent on the number of the source's antennas, a result that is in contrast to CSI-assisted relaying where the diversity order is equal to the minimum number of antennas between the source and the destination. Also, the case where the second hop is stronger reveals that the system's performance depends only on the antenna configuration at the source. The strong point of this work is that realistic assumptions are made and mapped to cases such as the uplink of a cellular network where antenna spacing causes correlation at the terminals or when two BSs communicate with the help of a singleantenna relay; that is, when these BSs have the same number of antennas, CSI-assisted relaying has a higher array gain and offers superior performance. In [37], the authors extend their previous published work [68] by examining the performance of two different relay protocols. The first is called channelnoise assisted (CNA) AF relaying that uses the statistics of the channel and the noise. The second protocol is called channel-assisted AF relaying and uses the statistics of the channels. The two protocols exhibit similar performance at high SNR. From the analysis, SER expressions are obtained and the diversity order and array gain for both protocols are extracted. The results indicate that antenna correlation is beneficial for cases where low SNR dominates the network while in the high SNR regime it is detrimental for the outage probability and SER. Finally, the achieved diversity order is equal to the minimum number of antennas between the source and the destination. The article in [69] studies a similar scenario with the above works, where a MIMO source communicates with a MIMO destination through a single-antenna relay. The analysis is given for two different systems. The first employs maximal ratio transmission (MRT) at the source and maximal ratio combining (MRC) at the destination and assumes general correlation structures. The second is based on transmit antenna selection (TAS) and assumes antenna correlation only at the destination. From the analysis closed-form expressions for outage probability, average symbol-error-rate (SER) and generalized higher moments of SNR are obtained for CSI-assisted and fixed-gain relaying. Also, a high SNR analysis is performed to gain an insight on the diversity of the system. It is concluded that CSIassisted relaying outperforms the fixed-gain and this holds for the MRT/MRC system in comparison to the TAS system.
In a different setup, the authors in [63] study a multipleaccess scenario where MIMO users want to communicate with MIMO BSs through MIMO relays. The antennas at all the nodes are considered correlated and modeled with Kronecker correlation. The challenge in this setup is that the BSs have perfect CSI while UEs and RTs have the channel covariance information (CCI). As a result, to maximize the system sum rate, the authors perform a joint optimization of the UEs covariance matrices and relay precoding matrices. From the analysis, the asymptotic sum-rate expression is derived for the large-system scenario where the antennas are increased in every network node with constant ratios. Moreover, results are obtained for various given signaling inputs, and an iterative algorithm is given which obtains the asymptotically optimal users and RS precoding matrices.

\section{Beamforming for Single-User Communications}

In this section, various works are presented that study MIMO $\mathrm{BF}$ techniques in cases where a single user is present in the network. Following, and for each scenario, an analytical description is performed evaluating the BF scheme.

3.1. Beamforming with Single Relay. In Figure 2, a network where a single relay is used to establish communication between a source and one user is depicted. Under the single-relay consideration and when capacity and power optimization arises, the authors in [45] provide the three basic modes for the three-terminal MIMO relay network, namely, the direct link (mode A), the relay without direct link (mode $\mathrm{B}$ ), and the relay with direct link (mode C). A weighting matrix at the relay is designed in such a way as to minimize capacity loss. This is achieved by transforming the MIMO relay channel in parallel SISO relay subchannels and then, through waterfilling, power allocation is performed.

The Grassmannian codebooks are proved appropriate for the design of the source and relay BF based on the distributions of the optimal source and relay $\mathrm{BF}$ vectors [34]. The authors aim at SNR maximization through BF at the source and the relay. The explored scenarios include an SRD topology with and without the presence of the SD link. Moreover, when perfect CSI is available at the source 


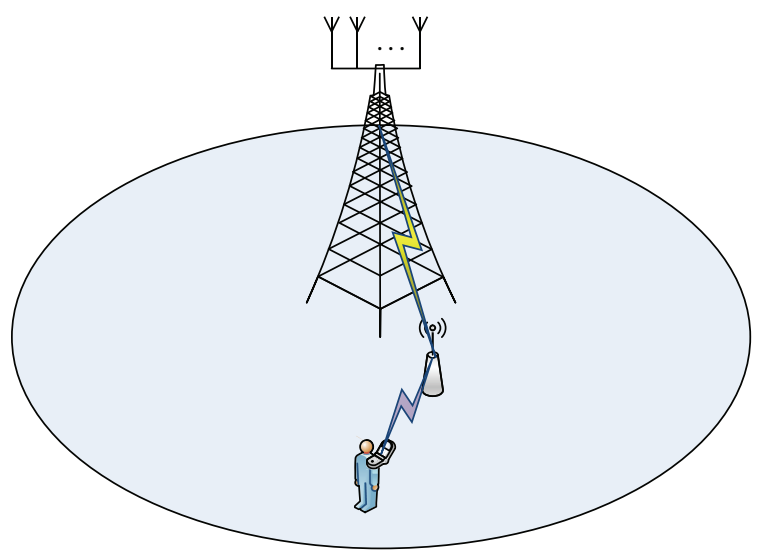

FIGURE 2: Single-relay communication.

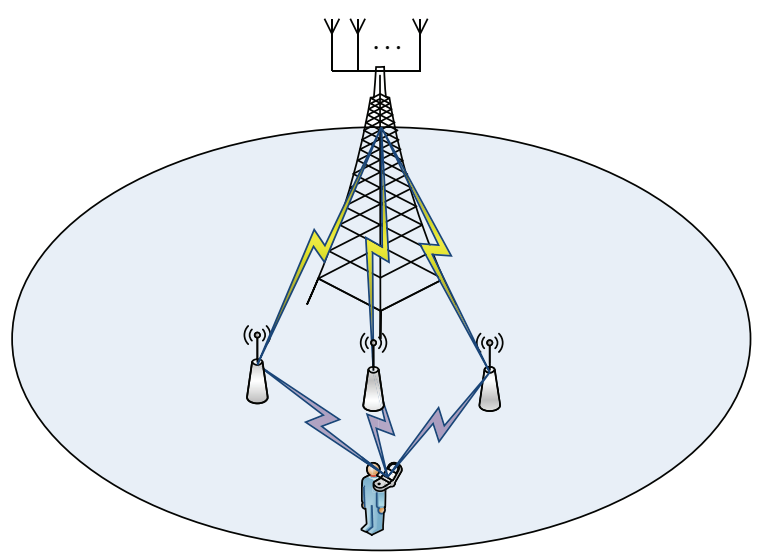

FIgURE 3: Multiple-relay communication. and the relay, a mapping of the source and relay signals to the dominant right singular vectors of the SR and $\mathrm{RD}$ channel should be performed. On the other hand, when limited feedback is available, Grassmannian BF codebooks at the source and the relay should be adopted which quantize the optimal BF vectors. In order to reduce the complexity, a modified quantized scheme can be employed for the case where SD connectivity is feasible. Through this scheme, only one singular vector needs to be quantized resulting in significant reduction of feedback from the destination to the relay.

The optimization (minimization) of the MSE in a singleuser single-relay scenario is considered in [23], where the authors consider the optimal BF in three stages. First, the MIMO relay performs receive $\mathrm{BF}$ by using the Hermitian transpose of the left singular matrix of the SR channel. Then, linear precoding takes place at the relay and, finally, transmit $\mathrm{BF}$ is performed through the use of the right singular matrix of the RD channel. The work in [25] extends the previous one by performing joint source-relay precoding design. As the derivation of the optimal relay amplification $\mathbf{F}$ and source precoding $\mathbf{B}$ matrices in closed form is intractable, an iterative algorithm is provided. The algorithm optimizes $\mathbf{F}$ for a given $\mathbf{B}$ under a relay power constraint. Also, B is optimized for a fixed $\mathbf{F}$ under power constraints at the source and the relay. Following the precoding technique, the authors in [24] propose a non-linear precoding scheme, which adopts a Tomlinson-Harashima precoder (THP) at the source, while the relay uses a linear precoder. Moreover, a direct SD link exists and the destination uses an MMSE receiver. The proposed scheme performs a joint sourcerelay precoder design and to make the solution tractable it decomposes the problem into one subproblem and a master problem, which provide the precoders of the source and the relay correspondingly. Comparison with the schemes of [21, 24] shows improved MSE performance from the proposed precoding method. Finally, the work in [70] studies the degrading effect of self-interference and provides precoding designs to mitigate it. More specifically, transmit and receive beams at the relay are formed in such a way to minimize the self-interference signal experienced at the receive antennas of the relay. This is achieved by pointing these beams to the minimum eigenmodes of the channel between transmit and receive relay antennas. Also, the authors provide the condition, which corresponds to the null-space projection scheme of the optimal eigen-BF, thus providing orthogonal subspaces to relay reception and transmission. The main contribution of this work is the formulation of precoding designs that allow the minimization of the degrading effects of self-interference.

3.2. Beamforming with Multiple Relays. Figure 3 illustrates the scenario where multiple relays are allocated for the communication between a source and one user. Moving forward and considering single-user BF for multiple-relay nodes, in [44], the authors compare signaling and routing techniques for various relaying protocols, namely, amplify-and-forward (AF), decode-and-forward (DF), and hybrid relaying, where the relay decodes only the necessary information to obtain CSI for the SR or RD channels. Several MIMO spatial multiplexing (SM) techniques are presented, which take advantage of CSI knowledge at the relay by coordinating the SR and $\mathrm{RD}$ channels eigenmodes. The authors compare SM to single signal BF (SSB), which exploits the spatial diversity of MIMO channels. If CSI is available at the transmitter, then SSB can be performed. In continuity, two cases of SSB are given for both DF and hybrid relaying. It is shown that, in the low SNR regime, SSB is preferable compared to spatial multiplexing due to its increased diversity. The main contribution of this article is the consideration of three types of relaying and the comparison of SM and SSB for various SNR regimes.

When a MIMO equalizer is introduced for implementing the BF matrix at the receiver, the authors in [21] study the SNR and MMSE designs under a global power constraint at the relays and at the receiver, in a network with MIMO source, multiple MIMO relays, and MIMO destination. For the MMSE approach, they provide two alternatives to formulate the relay matrix $\mathbf{F}$ and the MIMO equalizer $\mathbf{A}_{k}$ at the receiver. Firstly, they perform a two-step design where $\mathbf{F}$ is priorly formed and then $\mathbf{A}_{k}$ is derived. Secondly, they proceed in a joint formulation of $\mathbf{F}$ and $\mathbf{A}_{k}$. On the other hand, for the SNR 
approach, they include two optimization cases, with a zeroforcing constraint and with the global power constraint. Furthermore, they form the BF matrices aiming to maximize the transmission rate. The simulations show that both approaches achieve similar BER performance when there is no power constraint at the relays. By targeting SNR maximization at the destination under different power constraints, the authors in $[33,61]$ study a network where a MIMO source communicates with a single-antenna destination through multiple MIMO AF relays. Three different power constraints are derived for the optimal $\mathrm{BF}-\mathrm{AF}$ weights and for a given $\mathrm{BF}$ vector at the source. Firstly, for the individual and joint relay power constraints, closed-form solutions are given. Secondly, for the joint source-relay power constraint, a numerical method is presented which offers optimal power allocation for the source and the relays. In order to decrease implementation complexity, suboptimal methods for the joint relay and joint source-relay power constraints are presented. These methods are based on the transformation of the optimization problem into a nonconvex polynomial programming problem. Numerical results illustrate that the performance of the suboptimal methods follows closely that of the optimal one. By targeting minimization of the MSE or equivalently the maximization of the SINR under a sum power constraint, the study in [22] considers BF that is coupled with single-carrier frequency domain equalization in a three-node network. From the proposed algorithm, the optimal frequency-domain linear equalization (LE) and decision-feedback equalization (DFE) to the receivers is derived. The optimal relay BF matrices are formed under a sum power constraint. Moreover, complexity issues are considered by providing suboptimal power allocation algorithms without significant performance degradation.

3.3. Relay Selection. The case of relay selection is shown in Figure 4. In order to reduce synchronization requirements among the multiple transmitting relays, relay selection has been proposed in [10]. In addition, the selection of one relay or a subset of the available relays improves the spectral efficiency of the transmission as the amount of orthogonal channels is less than the number of the relays in the network for the same diversity gain. In [35], the authors illustrate the efficiency of relay selection through comparisons with BF schemes based on limited and unlimited CSI. They develop the outage probability of the optimal AF BF with unlimited feedback noting that due to its impractical assumptions it serves only as a performance bound to other more practical schemes. Moreover, the selection scheme is proven to be the unique optimal for $\mathrm{AF} \mathrm{BF}$ as it minimizes noise amplification. Also, numerical comparisons are given for the cases of optimal codebook design and random BF with limited feedback. The compared schemes include relay selection, optimal BF, and BF with various amounts of feedback. It is concluded that the selection scheme outperforms the other BF schemes in limited feedback scenarios while alleviating synchronization concerns.

Partial relay selection (PRS) is employed in [36], where suboptimal relay selection is presented, as the only CSI

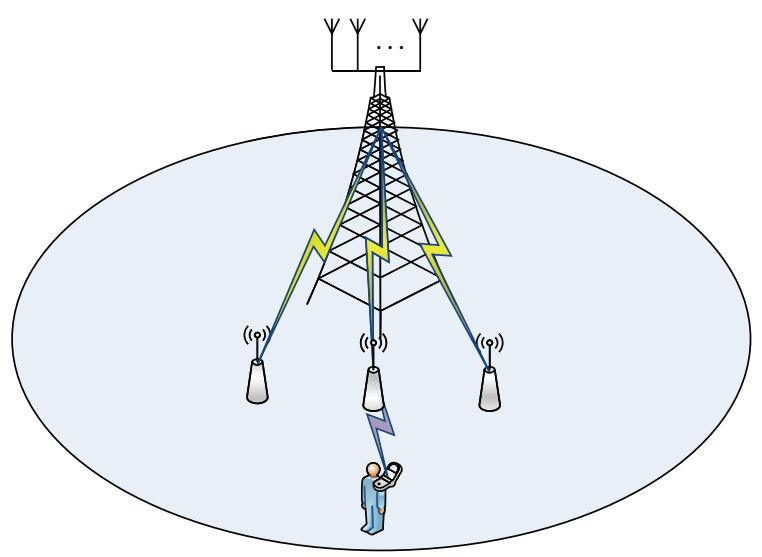

Figure 4: Communication with relay selection.

considered in the selection algorithm is the quality of the SR links. Furthermore, transmit and receive BF is implemented at the source and the destination, while for the selected AF MIMO relay the linear precoder is optimized. Additionally, the outage probability of the proposed scheme is given in closed form and for the asymptotically high SNR the diversity gain is extracted. As a result, PRS and OR achieve the same diversity gain.

Another technique is the opportunistic selection of a semiorthogonal subset of relays [47]. The proposed scheme takes place in two steps. First, spatial eigen-mode combining between the forward and backward channels is performed. Then, for the selected subset, the algorithm proceeds in antenna pair selection for reception in the first hop and transmission in the second hop.

The best relay selection is studied in [62], where a singleuser network is examined with the consideration of multiple MIMO relays. The proposed scheme selects the best relay that successively combines maximal ratio receiver combining in the first hop and BF in the second. In order for this process to take place, the authors consider that in two sequential time slots the channel coefficients remain constant. Simulations include comparisons with various relay numbers and antenna numbers on each relay, illustrating the reduction of the outage probability as these values increase. Along with the best relay selection, there are other selection techniques that incorporate, for example, max-rate selection with interference mitigation issues. The work in [46] considers a multihop backbone network where MIMO relays are employed. In each phase, a relay is selected based on maximum rate path routing and performs transmit BF. Additionally, mitigation of the multiple access interference that degrades the performance of the network is achieved through cancellation. The effects of interference mitigation transmit $\mathrm{BF}$ and spatial reuse on the performance of the proposed scheme are studied in a game theoretic approach, aiming at the optimal combination of these techniques.

Finally, the multi-relay network of [71] selects in each time slot two relays in order to achieve full-duplex operation through successive relaying. To this end, buffer-aided relay selection is combined with beamforming and two schemes 
are proposed. The first scheme is inspired by the case of no IRI between the relays and adopts MRC at the receiving relay and MRT BF at the transmitting relay. As IRI is considered, this scheme utilizes an SINR criterion for the SR link and the relay-pair that maximizes the instantaneous end-to-end rate is chosen. The second scheme is based on ZF-BF to cancel the IRI at the receiving relay and to maximize the effective channel power gain of the RD link. Results illustrate that the SINR-based approach improves the rate performance of the network in the low SNR region while the ZF scheme has the best performance and approaches the upper bound of the IRIfree case.

\section{Beamforming for Multiuser Communications}

An alternative approach for transmitting the signal through the relays is to serve multiple users simultaneously. A relay broadcast channel (RBC) can be considered, which is a typical case for the so-called nondedicated relay system. A nondedicated relay system is when the mobile users can help each other by relaying information for their peers besides receiving their own data. An $\mathrm{RBC}$ is based on a broadcast channel (BC) where a BS transmits to multiple users simultaneously. Relay mechanism is presented into the $\mathrm{BC}$ in such a way that the users can benefit from each other by performing cooperative procedures. In addition, another case considered is that of two-way relaying as a multiuser scenario, where the two sources exchanging information could be single or multiple pairs of users that communicate and not necessarily a BS and a user.

There are several ways of exploiting this operation by employing MIMO techniques, as depicted in Figures 5-7. Several scenarios can exist, such as multiuser communication through a single relay, multiuser communication through multiple relays, two-way single pair communication, and two-way multi-pair communication.

4.1. Beamforming with Single Relay. When a single relay is concerned, as is the case in Figure 5, there are many studies that take into consideration some or all of the aforementioned challenges discussed in Section 2. Different power constraints, precoding techniques, and feedbacks are introduced to minimize MSE, maximize sum rate, and decrease complexity. The classification of the examined papers depends on whether users are equipped with multiple antennas or not.

On one hand, when only a MIMO BS and a MIMO RS are considered, a solution for the optimization problem is introduced in [49], which exploits the downlink performance, incorporating relay $\mathrm{BF}$ with source precoder matrix. It deals with a formation of MIMO relaying broadcast channel to multiple users at the downlink, based on weighted sum-rate criterion. Accordingly, the design of the source and relay matrices is based on non-linear and nonconvex Weighted MMSE (WMMSE) criterion. The WMMSE scheme can better deal with the multiuser interferences and noise, and the relation between downlink gains and source-relay

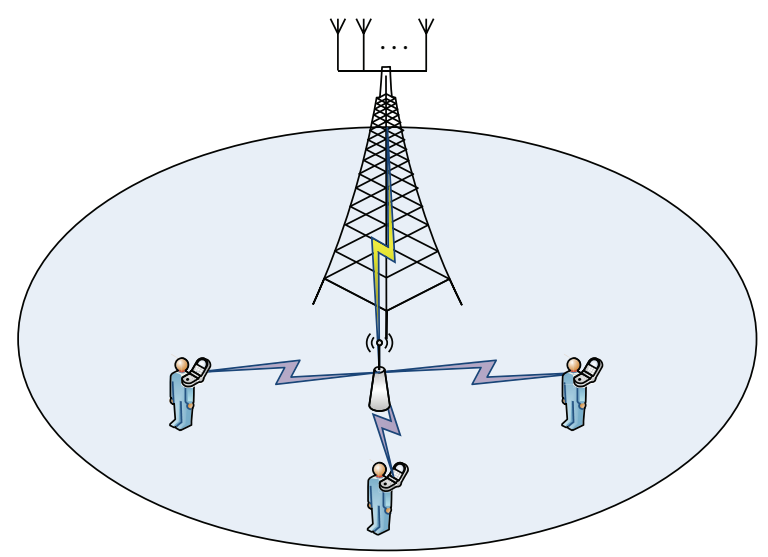

Figure 5: Multiuser communication through a single relay.

users channel gains. Because of the difficulty in solving the non-linear and nonconvex problem, decoupling it into two tractable subproblems solves an equivalent problem and an alternative optimization based on efficient linear iterative design algorithm is proposed, which always converges to a stationary point. Following the previous topology, the authors in [40] consider a MIMO relay-assisted multiuser downlink transmission with limited feedback and suggest two precoding schemes at the RS based on the ZF criterion and the MMSE criterion. These robust linear BF schemes take only channel direction information (CDI) feedback using a finite number of feedback bits to the RS and the effect of channel quantization errors for determining the BF vectors.

On the other hand, when all nodes have multiple antennas, the authors in [39] consider MIMO relay broadcast channels. For simplicity, a two-user system is taken into consideration. A precoding, relaying, and combining (cooperative) scheme is proposed, under an overall power constraint, and an optimal power allocation solution in a closed form is developed. Instead of considering time-division broadcast schemes, it is assumed that the BS transmits simultaneously to both users in the same frequency band. Precoding is used to steer the signals for the two users in different subspaces to avoid interuser interference, employing the zero-forcing criterion. The user with better conditions acts as an AF relay to the other user besides receiving its own signal. Based on the proposed scheme, an optimal power allocation solution is established so as to minimize the BER, which is equivalent to maximize the SNRs. Moreover, an optimization algorithm for the BF vectors is proposed in order to achieve the maximum effective channel gains, utilizing three different schemes for optimization of combining vectors: exhaustive search, blind algorithm, and broadcasting optimization. The proposed algorithm is shown to achieve a near optimal performance (compared with the exhaustive search algorithm) and maximum diversity gain. Nevertheless, there are several weaknesses: only a fixed relay direction has been considered, the $\mathrm{BF}$ and combining vectors have been determined in sequence, which is not optimal, and only one data stream for each user and flat fading channels have been taken into consideration. 


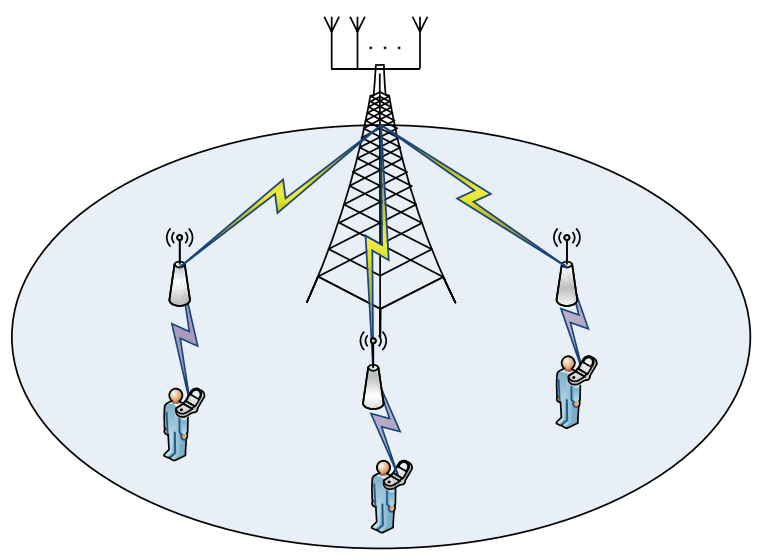

Figure 6: Multiuser communication through multiple relays.

Accordingly, a fully MIMO single-relay scheme is presented in [48] where the topology comprises a MIMO BS, where a dirty paper coding is applied, a fixed infrastructurebased half-duplex MIMO relay station (RS) with linear processing, and multiple users equipped with multiple antennas. A MIMO BRC and its dual multiple access relay channel (MARC) network (uplink-downlink duality) is used to transform the problem and solve a nonconvex problem which finds the input covariance matrices and the RS BF matrix that maximize the system sum rate. To make the problem tractable, the relay $\mathrm{BF}$ to the left and right singular vectors of the forward (RS-to-users) and backward (BS-to-RS) channels was matched. With this RS BF structure, the authors proposed an iterative algorithm for the sum-rate maximization for the dual MIMO MARC, where the MIMO-AF duality was proved for multiple-antenna-user networks. The proposed scheme follows an alternating-minimization convergent procedure over the input covariance matrices at the transmitter and the $\mathrm{BF}$ matrix at the relay. Also, the derivation of the mapping from the resulting covariance matrices for the MARC to the desired covariance matrices for the BRC is proposed. A valuable observation for better system performance is to have more antennas at the RS than at the BS.

Following the consideration of a fully MIMO singlerelay topology, a linear BF design for amplify-and-forward relaying cellular networks is considered in [26]. The design is based on optimizing (minimizing) the sum mean square errors of multiple data streams, while joint design of the precoders, forwarding matrix, and equalizers for both uplink and downlink is considered and under individual power constraints. An iterative algorithm is proposed for the downlink so as to jointly design the precoder at BS, while forwarding matrix at RS and equalizers at mobile terminal. For the uplink, the duality of the BF design is demonstrated and the same downlink iterative algorithm can be applied. Additionally, a low-complexity algorithm has been developed for the uplink under a special case when the number of independent data streams from different mobile terminals is greater than or equal to their number of antennas (fully loaded or overloaded uplink systems). It is found that the resultant solution includes several existing algorithms for

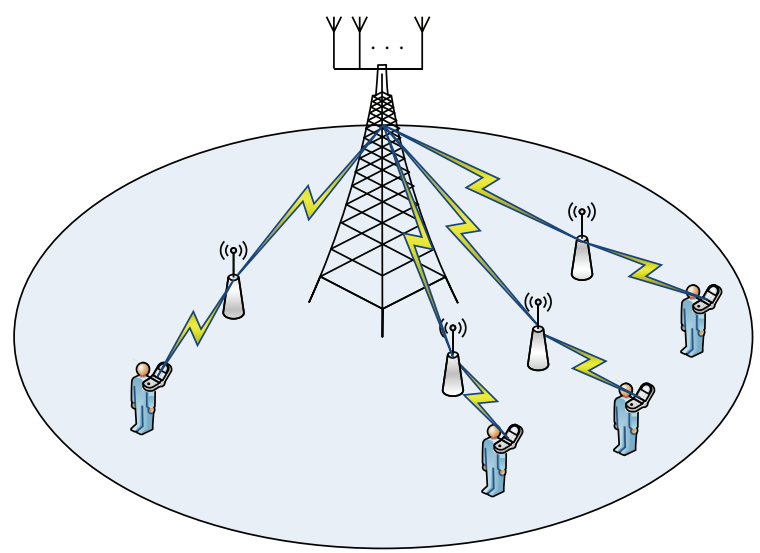

Figure 7: (Left) Two-way single pair communication, (right) twoway multi-pair communication.

multiuser MIMO or AF relay network with single antenna as special cases and outperforms the suboptimal schemes. It is verified that for AF MIMO relaying systems, source precoder design is of great importance and offers additional design freedom for performance improvement.

Finally, when both single- and distributed-relaying schemes are considered and compared in [43], multiple single-antenna SD pairs communicate via one MIMO relay in a network where linear BF techniques are employed. The goal of $\mathrm{BF}$ is the sum-power minimization aiming at a target SINR at the destinations. The BF technique is based on ZF in order to cancel the interference at the destinations and the formulation of the relay BF matrix result in a least-squares problem that can be solved with convex optimization tools. From the numerical results, it is concluded that the MIMO relay with ZF-BF achieves the best performance compared to other schemes employing distributed single-antenna relays.

4.2. Beamforming with Multiple Relays. Interference management is maybe the most fundamental open problem in wireless networks. When multiple MIMO relays are concerned, as shown in Figure 6, thus enhancing the SD pairs equipped with multiple or single antennas, and incorporate beamforming techniques for throughput improving, interference issues appear. The following studies are inspired by this problem and confront the arising interference issues by introducing beamforming algorithms under different network topologies.

In regard with the first topology, where multiple SD pairs communicate through multiple MIMO AF relays, the authors of [42] develop two adaptive relay $\mathrm{BF}$ algorithms employing linearly constrained $\mathrm{BF}$ algorithms with minimum variance based on Kalman filtering. As a result, the received power at the destinations is minimized by considering the linear constraints on the relay BF matrices, thus avoiding severe degradation of the reception by noise and interference while preserving the desired signal at each destination. The main differentiation of these algorithms is that the first operates in a centralized fashion while the second is distributed. In the centralized approach, the relays have a common processing 
center that receives all the CSI and computes the BF coefficients, which are fed back to the relays. In the distributed case, each relay computes its own BF coefficients through local channel estimation. Additionally, extensions to these algorithms are discussed through power control and QoS modification. Numerical results indicate similar performance of the proposed methods to the noniterative centralized second-order cone program (SOCP)-based algorithm at low and medium SNR regimes and with reduced computational complexity. The main contribution of this work is the reduced complexity of the two proposed algorithms compared to the SOCP-based BF algorithm and the formulation of a distributed BF algorithm.

Following the first topology, there are several papers dealing with a new cooperative interference management scheme named interference neutralization. Accordingly, in [55], illustrative examples are discussed in a network consisting of multiple sources, relays, and destinations. This technique is based on the concept of neutralizing the interference signal at the destination provided that this interference is propagated through various paths. In practice, these interfering signals are processed so that they have the same power level and neutralize each other by adding them at the destination. This processing can take place at the relay using a proper permutation. In addition, the use of lattice codes to achieve the neutralization effect is shown and the signal received after the summation of the interfering signals is the desired point on the scaled lattice. The main contribution of this work is the detailed description of interference neutralization. In [56], authors deal with the mitigation of the cochannel interference issues that arise when relays are equipped with multiple antennas operating in AF and half-duplex modes. Selecting a pair of multiple sources and multiple destinations, both of which are equipped with single antenna, enhances the network performance. A coordinated relay beamforming is considered to suppress interference and improve the date rates of two-hop interference networks, under the sum-rate maximization criterion. A suboptimal solution of interference neutralization beamforming is then introduced, which allows the interference to be canceled over the air at the last hop, where the relay beamformer is designed to neutralize interferences at each destination terminal.

Concerning the second topology, where a number of MIMO half-duplex relays aid the data transmission from a number of transmitters MIMO BSs to their associated MIMO users, the study in [50] conceived an interference management approach. This approach considers interference broadcast channels at relays and end users, where a number of MIMO half-duplex DF relays aid the data transmission from a number of transmitters MIMO BSs to their associated MIMO users. A typically linear precoding design at the transmitter and $\mathrm{BF}$ matrix at the relay is accomplished, so as to maximize the end-to-end sum rates. The proposed algorithm solves in a suboptimum way the transmit precoder design following three phases: (1) second-hop transmit precoder design, where the relays are designed to maximize the second-hop sum rates; (2) first-hop transmit precoder design, where approximate end-to-end rates that depend on the time-sharing fraction and the second-hop rates are used to formulate a sum-utility maximization problem to design the transmitters; this problem is solved by iteratively minimizing the weighted sum of mean square errors; (3) first-hop transmit power control, where the norms of the transmit precoders at the transmitters are adjusted to eliminate rate mismatch. The second hop is treated as the conventional single-hop interference broadcast channel and existing single-hop algorithms can be applied to find the stationary points of second-hop sumrate maximization. The design of the first-hop precoders is devised by applying a naive approach ignoring the designed second-hop transmit precoders. The overall performance is subjected to the assumptions of each transmitting node (relay and BS) and has instantaneous and perfect local channel state information (CSI) and there is a feedback channel to send information from a receiving node to its serving node. Finally, the algorithm is implemented in a quite reverse mode; the relays are optimized for second-hop sum-rate maximization before the transmitters are designed for end-to-end sum-rate maximization. An interesting interference pricing scheme is employed in [57], where the authors investigate the twohop interference channel and map it to a cellular network with relays. Interference pricing is employed to allow the relays to take into consideration the impact of interference on the end-to-end rate. In order to avoid rate mismatch in the two-hop transmission, an approximation is proposed in the computation of the end-to-end achievable rate, which incorporates the interaction of the two-hop channels, that is, which hop is dominant.

4.3. Two-Way Communication. In Figure 7, two-way relaying scenarios are shown for single and multiple pairs. Two-way communication considers two source nodes that exchange their information through an assisting relay node. When beamforming algorithms are engaged at the exchange scheme, the delivery of information can be completed in two time slots. In the first time slot, both source nodes simultaneously transmit signals to the relay node. In the second time slot, the relay node precodes the received signals along with various beamforming techniques and broadcasts the signals to both source nodes. Self-interference and cointerference issues arise and several multiple access and network coding techniques have been proposed for optimization of a twoway relay channel (TWRC) communication system. When one relay is dedicated to a single user, then a single-pair SD is accounted for, whereas when multiple users utilize one relay, a multiple-pair SD is taken into consideration.

4.3.1. Single Pair. The first set of papers deal with precoding at the relay node. In [32], the authors focus on designing the precoders and decoders based on the MSMSE criterion in a topology where multiple MIMO relays assist two MIMO sources. To simplify the optimization process, the decomposition of the primal problem into four subproblems is performed. These problems include the derivation of the optimal decoders at the sources, and the optimal relay precoding matrix, the optimal precoding matrix for the first source and then for the second source. It is noted that the solution of the second subproblem is one of the 
main contributions of this work as it is converted into a convex problem that can be solved. Also, a simulation setup of the proposed scheme is presented and comparisons with suboptimal versions and a nonprecoded scheme are performed. In [29], the main task is the derivation of the optimal structure of the precoding matrices at the sources and the MIMO relay, when MMSE receivers are used as they reduce the complexity in comparison to joint $\mathrm{ML}$ detection. Since the joint optimization problem is proven to be nonconvex (Schur-concave/Shur-convex), an iterative algorithm is developed to find the optimal precoding matrices. The algorithm is initialized by finding a simplified relay precoding matrix, designed for the cases where relay antennas are twice (or greater) the number of the antennas of each source. Afterwards, for fixed precoding matrices at the relay and one source, the optimal matrix at the other source is designed. Finally, joint optimization can be performed by updating the relay precoding matrix with fixed matrices at the source and then update the sources' precoding matrices with a fixed relay matrix. Numerical results show the efficiency of the proposed algorithm in terms of normalized MSE and BER and with significantly lower complexity when the suboptimal relay matrix is selected.

Along with the precoding strategy, various network coding schemes found prolific field for increasing the spectral efficiency of the network, alleviating interference issues, and thus optimizing the single-pair BF performance. The work in [58] presents a three-node topology where the end nodes communicate through a MIMO relay. The relay follows a network coding strategy based on either digital network coding or physical network coding. As a result, the precoding strategy in the broadcast phase takes into consideration the maximization of the minimum distance of the networkcoded symbols. For this reason, a hybrid precoder is proposed which switches among three suboptimal precoders, that is, with subspace alignment, with subspace separation, and with maximum ratio transmission. Numerical results include comparisons of the three suboptimal precoders with the hybrid precoder and a reference scheme where each end node does not cause interference to the reception of the other node's signal at the relay. The result proves the efficiency of the proposed scheme as it achieves a near-optimal frame error rate (FER) performance. The work in [54] studies a threenode topology with single-antenna source and a MIMO AF relay. To increase the spectral efficiency of the network, analogue network coding is performed which results in the cancellation of the self-interference at the sources caused by the previously transmitted messages. The authors derive the optimal BF matrix at the relay through SVD as well as its achievable capacity region. The capacity limits are extracted through the use of rate profiles, which regulate the ratio of the rate of a user to their sum rate as a predefined value. In addition, power minimization is performed at the relay given specific SNR at the receivers. In order to offer more practical schemes for this topology, two suboptimal approaches based on matched filter and ZF are presented. The results indicate that the matched filter approach is the scheme, which can offer the best performance considering its lower complexity compared to the optimal BF technique. In
[30], relay selection is combined with two-way transmissions in a network consisting of two single-antenna sources that are assisted by $K$ MIMO relays. The optimal relay selection criterion is extracted by considering the minimum PLNC decoding error probability. To perform the selection, each relay transformation matrix is decided according to [54] and then the one that provides the minimum decoding error probability is selected. Numerical results show the diversity gain achieved by relay selection through the proposed criterion.

A sum-rate maximizing technique is proposed in [53], which performs joint optimization at the relay. The authors aim at the maximization of the sum rate in a two-way communication scenario with a MIMO relay. To this end, a sumrate maximizing technique is proposed which performs joint optimization at the relay. As the derivation of the optimal processing matrix is nonconvex, an approximate solution is proposed which consists of the iteration of three optimization problems for the transmit beamformer, the receive combiner, and the linear relaying matrix. Simulations compare the proposed scheme with other single and multiple antenna techniques in terms of sum-rate performance. It is concluded that the upper bound is achieved by the proposed BF scheme while it converges rapidly to the final solution. Alternatively, when the minimization of the sum mean square error (SMSE) is evolved, the authors in [31] jointly optimize the ideal BF vectors for the two communicating sources and the MIMO relay with the target of SMSE minimization, under individual power constraints at all the nodes. A simplification analysis is then conducted stating that the BF pairs, which minimize the SMSE, also maximize the SNR at both communicating nodes. In addition, a scheme, which optimizes the BF vectors in three consecutive steps, is given and is compared to the optimal one in terms of the number of iteration and BER. Results indicate that when the number of antennas is greater than two, the simplified scheme experiences only a small performance loss and can substitute the optimal one.

4.3.2. Multiple Pairs. Considering the multiple-pair twoway communication scheme, numerous works exist that have tried to improve all the above-mentioned challenges in Section 2. Regarding the channel estimation challenge, the authors in [51] consider multiple SD pairs that communicate through MIMO AF relays and adopt the two-way strategy. The nodes are assumed to be full-duplex and perfect CSI is available to all of them. In this context, two different cases are presented. The first is termed $Y$ relay channel and consists of three users that want to unicast independent messages for different two users through the relay. For this setting, the achievable degrees of freedom (DoF) are extracted when the relay performs either analog network coding (ANC) or physical layer network coding (PLNC) and are proven to be equal to $2 M$ if all the nodes have $M$ antennas. The second case considers multiple two-way relay links that operate concurrently, thus naming this case as two-way relay $X$ channels. By equipping the users with $M=3$ antennas and the relay with $N=4$ antennas, the DoF is found to be equal to 8 . The main contribution of this article is the investigation 
of the DoF in two-way relay networks. Correspondingly, the formulation of a general model for topologies where $N$ single-antenna nodes communicate simultaneously ( $N$-way) through a MIMO relay is presented in [64]. From the analysis it is extracted that for this general case there should be at least $N-1$ antennas at the relays while the transmission should occupy $N$ time slot. Moreover, the general BF matrix at the relay is constructed for various objective functions.

An interesting relaying scheme termed Quantify-andForward (QF) is considered in [41], where the authors investigate a topology consisting of $K$ single-antenna pairs that perform two-way communication with the help of a MIMO relay, which is equipped with $M \geq 2 K$ antennas. The relaying strategies include $\mathrm{AF}$ and $\mathrm{QF}$ and the corresponding $\mathrm{BF}$ techniques are derived. For AF relaying, two possibilities are considered; the first is $\mathrm{BF}$ with $\mathrm{ZF}$ transmitter and receiver and the second performs block diagonalization, which takes into consideration that intrapair interference can be cancelled. Also, the QF strategy, which can be seen as a case of analog network coding due to the signal separation, is performed at the relay. Furthermore, the received signals at the relay are quantized to a scalar that is a linear combination of the two-dimensional vectors transmitted by each pair. In the next phase, multicast aware BF is employed aiming to minimize distortion. Additionally, comparisons are performed between the proposed schemes and DF relaying in terms of the average sum rate showing improved performance in a wide range of SNRs. The main contribution of this work is the consideration of $\mathrm{AF}$ and $\mathrm{QF}$ strategies that do not require the knowledge of the codebooks of the mobiles by the relay. Also, through the QF scheme, simpler signal representation is achieved through signal separation at the relay.

The challenge of power minimization is the subject of [28]. The article studies a topology where $2 \mathrm{~K}$ MIMO users communicate in pairs through a MIMO AF relay. The optimization problem is formulated for both $\mathrm{ZF}$ and MMSE criteria taking into consideration a power constraint at the relay and predetermined transmit-receive $\mathrm{BF}$ vectors which can be obtained from CSI. Also, four BF methods are presented which deal with different CSI conditions; firstly, eigen-BF that requires perfect CSI knowledge at the users in order to produce the eigen-BF vector; secondly, antenna selection that can be performed with partial CSI as only the CSI of the selected antenna is fed back to the relay; thirdly, random $\mathrm{BF}$ which does not assume CSI knowledge but needs synchronization information among the users and the relay; finally, equal gain BF that also does not need any CSI and no further information at the relay. Another area that the authors investigate is the power control for ZF systems. Two different cases are studied starting with local power control that distributes the multiuser power to the users according to their channel conditions and then global power control that for the high SNR regime divides network power to the users and the relay with the target of system SNR maximization. All the proposed BF methods are evaluated through simulations illustrating the tradeoff between improved performance and CSI overhead. The main contribution of this paper is the investigation of four different BF methods that can be chosen according to the availability of CSI in the network.
In [52], physical layer network coding is proposed in a topology where one MIMO BS with $M$ antennas communicates with $M$ single-antenna mobile stations through a MIMO relay that also has $M$ antennas. The communication protocol is based on two-way relaying in order to enhance the spectral efficiency of the network. The BF method is based on interference alignment that aims to put the two messages, which are transmitted and received by each user, in the same spatial direction at the relay. In this way, cochannel interference, that is, the major degrading factor in this network, is mitigated. Moreover, the precoding designs for the matrices at the BS and the RS are given in detail, under individual power constraints and the minimization of CCI. Through analytic results, it is proven that the multiplexing gain achieved is equal to the number of the mobile stations while the diversity gain can be increased by employing multiple MIMO relays and by increasing the number of antennas at the BS and RS to be greater than $M$. Finally, simulations are performed to compare the proposed network coded scheme to time sharing network coding approaches, thus illustrating the improved performance of this scheme. The main contribution of this work is the interference alignment inspired network coding technique and the discussions of multirelay and increased antenna number cases.

Finally, while aiming to optimize the total MSE and sum rate and combat interference, different precoding schemes are applied for enhancing the uplink transmission performance [59]. This work investigates two-way relaying for a topology where a MIMO BS with an ML decoder, communicates with $K$ single-antenna users through a common MIMO AF relay. The objective is to maximize the minimum symbol distance at the BS, that is, to improve the uplink performance. Moreover, three different precoding cases are presented which require varying complexity and a clean relay model that assumes negligible noise at the relay is introduced as well. Firstly, precoding at the BS is considered while the relaying only amplified the received signal under the imposed power constraint. Secondly, precoding at the RS under the aforementioned optimization target is proven to be nonconvex and to obtain a solution the transformation into another optimization problem is given which can be solved through bisection search to acquire the quasioptimal solution. The third precoding design considers joint precoding at the BS and the RS to further improve the system's performance. These precoding methods are compared via simulation and the joint scheme shows the best performance but at the cost of increased complexity in its practical implementation. Through the proposed methods, self-interference and cochannel interference are efficiently mitigated. The authors in [27] extend the study in [59] by considering a similar cellular multiuser two-way relaying topology and aiming to optimize the total MSE and sum rate. Linear precoding designs are given for BS precoding, RS precoding, and joint BS-RS precoding.

\section{Discussion and Open Issues}

This section provides a discussion based on the works that were presented throughout this survey and, in addition, some 
TABLE 2: Classification of articles based on network topology, the optimization target with the corresponding power constraint, and the relaying topology.

\begin{tabular}{|c|c|c|c|c|}
\hline $\begin{array}{l}\text { Reference } \\
\text { article }\end{array}$ & Network topology & Optimization target & Power constraint & Relaying topology \\
\hline$[21]$ & Single-user & MSE, SINR, Capacity & Relay (total), receiver (interference level) & Multirelay \\
\hline$[22]$ & Single-user & MSE & Relay (sum-power) & Multirelay \\
\hline [23] & Single-user & MSE & Relay & Single-relay \\
\hline$[24]$ & Single-user & MSE & Source-relay (separate) & Single-relay \\
\hline [25] & Single-user & MSE & Source-relay (separate) & Single-relay \\
\hline [26] & Multiuser & MSE & Source-relay (separate) & Single-relay \\
\hline [27] & Two-way multipair & MSE & Relay & Single-relay \\
\hline [28] & Two-way multipair & MSE, SINR & Relay & Single-relay \\
\hline [29] & Two-way single-pair & MSE & Sources-relay (separate and individual) & Single-relay \\
\hline$[30]$ & Two-way single-pair & MSE & Sources-relays (separate and individual) & Relay selection \\
\hline$[31]$ & Two-way single-pair & MSE & Sources-relay (separate and individual) & Single-relay \\
\hline [32] & Two-way single-pair & MSE & Sources (individual)-relays (sum-power) & Multirelay \\
\hline [33] & Single-user & SNR & Source-relay (joint) & Multirelay \\
\hline$[61]$ & Single-user & SNR & $\begin{array}{l}\text { Source (individual)-relay (total), relay } \\
\text { (joint and individual) }\end{array}$ & Multirelay \\
\hline [34] & Single-user & SNR & Source-relay (separate) & Single-relay \\
\hline [35] & Single-user & SNR & Source-relay individual & Relay selection \\
\hline [36] & Single-user & SNR & Source-(selected) relay (separate) & Relay selection \\
\hline [37] & Single-user & SNR & Relay & Relay selection \\
\hline$[38]$ & Multiuser & SNR & Source-relay (joint) & Single-relay \\
\hline$[39]$ & Multiuser & SNR & Source-relay (joint) & Single-relay \\
\hline$[40]$ & Multiuser & SINR & Relay & Single-relay \\
\hline$[41]$ & Two-way multipair & SINR & Sources-relay (separate and individual) & Single-relay \\
\hline$[42]$ & Multiuser & SINR & $\begin{array}{l}\text { Relay (individual), receiver (interference } \\
\text { level) }\end{array}$ & Multirelay \\
\hline [43] & Multiuser & SINR & Relay (sum-power) & Multirelay \\
\hline$[44]$ & Single-user & Capacity & Relay (sum-power) & Multirelay \\
\hline [45] & Single-user & Capacity & Relay & Single-relay \\
\hline [46] & Single-user & Capacity & Relay & Relay selection \\
\hline$[47]$ & Single-user & Capacity & Sources-relays (separate sum-power) & Relay selection \\
\hline$[48]$ & Multiuser & Capacity & Source-relay (separate) & Single-relay \\
\hline [49] & Multiuser & Capacity & Source-relay (separate) & Single-relay \\
\hline$[50]$ & Multiuser & Capacity & Sources-relays (separate sum-power) & Multirelay \\
\hline$[51]$ & Two-way multipair & Capacity & Relay & Multirelay \\
\hline [52] & Two-way multipair & Capacity & Relay & Single-relay \\
\hline$[53]$ & Two-way single-pair & Capacity & Sources-relay (separate and individual) & Single-relay \\
\hline [54] & Two-way single pair & Capacity & Sources-relay (separate and individual) & Single-relay \\
\hline [55] & Multiuser & Capacity & Sources (individual)-relays (individual) & Multirelay \\
\hline [56] & Multiuser & Capacity & Relays (sum-power) & Multirelay \\
\hline$[57]$ & Multiuser & Capacity & Receiver (interference levels) & Multirelay \\
\hline$[58]$ & Two-way single-pair & Minimum symbol distance & Sources-relay (separate and individual) & Single-relay \\
\hline$[59]$ & Two-way multipair & Minimum symbol distance & Sources-relay (separate and individual) & Single-relay \\
\hline
\end{tabular}


open research topics that are of great interest and have not yet been sufficiently examined by the community. Table 2 depicts the references that were presented in the context of this survey. More specifically, each article is classified based on network topology, the optimization target with the corresponding power constraint, and the relaying topology that was employed. In general, most works investigate beamforming with half-duplex relays to avoid self-interference but the performance of the network is limited by the halfduplex constraint. Single user network has been a very active research field as it is a simple communication paradigm that allows the proposed BF techniques to clearly expose their operation. It is observed that a lot of contributions have been made in the two-way communications, as it is a strategy that improves the spectral efficiency and can provide significant gains, if the interference between the communicating end nodes is efficiently mitigated. On the other hand, as it is also the case with one-way multiuser communications, relay selection has not been considered as an alternative cooperation strategy and this offers a possible research direction towards complexity reduction. From the optimization target's perspective, there are numerous works that aim at MSE minimization, SNR, or SINR increase and capacity improvement. As network-coding algorithms combined with beamforming have recently been developed, there are a few works, which aim at the maximization of the minimum distance of network-coded symbols.

Although the area of BF with MIMO relaying has seen a significant number of contributions, there are many open issues that need to be investigated in the future. An important parameter that has to be taken into consideration is the synchronization among the various network nodes, especially in topologies where multiple relays are employed. More specifically, synchronization based on consensus algorithms [72] and single-hop on-off keying orthogonal signaling technique [73] inspired by sensor networks, distributed solutions such as those proposed in the context of relay selection [10, 74] should be adjusted, so as to satisfy the needs of networks that implement BF.

An overall requirement for the efficient implementation of BF techniques is CSI. As the majority of studies in the field examine schemes where perfect CSI is assumed, there is a lot of research to be done for practical schemes that will be robust when CSI is partially available, or impairments such as delay and channel estimation uncertainties affect its exploitation. Moreover, distributed solutions must be developed that will make use of local CSI knowledge, as is the case in [36], and formulate accordingly the BF matrices based on partial state information. These limited CSI cases could be extended to other network topologies, such as broadcast channels and full-duplex relaying schemes, which are discussed subsequently. The exploitation of CSI is also studied in [75], which addresses the optimization problem for a three-hop wireless network, where collaborative AF relaying terminals appear at both the transmitter and receiver ends to form a virtual MIMO system. This work is a step towards extending previous published results, which considered collaborativerelay beamforming (CRBF) only on one side, giving rise to a dual-hop communications system.
Moreover, recently there has been an increased interest in full-duplex relaying. Novel BF techniques should benefit from the increased spectral efficiency of this relaying scheme. $\mathrm{BF}$ algorithms should consider the loop interference among the receive and transmit antennas of the relay and find ways to mitigate it, appropriately forming the precoding matrix at the source and the BF matrix at the relay. Further schemes based on half-duplex relays but aiming at recovering the half-duplex loss are two-way and successive relaying. In networks where two-way relaying is applied to improve spectral efficiency, network coding approaches have started to receive significant contributions $[52,54,58]$, but there is enough space for additional work in this area. For successive relaying topologies, only [71] has proposed BF techniques and there is increased interest in this field for further research as the half-duplex loss can be recovered. Successive relaying networks perform concurrent transmissions by the source and one transmitting relay. As a result, interrelay interference arises and the BF matrix at the relay could be structured in such a way to minimize IRI, while achieving the performance target at the RD link. Likewise, the source precoding matrix should aim at increasing the SINR at the receiving relay, thus offering increased protection to the IRI from the transmitting relay.

Another field that has not been sufficiently researched until now is the BF designs for cognitive relay networks, where only few works have considered such topologies. In [76], various relay BF algorithms are proposed in a network where primary and secondary users coexist. BF aims at interference minimization to the primary users and rate maximization for the secondary users through iterative algorithms. Also in [77], the authors propose cognitive MIMO relay selection to maximize the capacity of the secondary user and by employing BF the interference to the primary user is minimized. As the exploitation of spatial resources is at the heart of BF, an adaptation of such a technique for networks consisting of primary and secondary users would provide additional gains in spectral efficiency. Moreover, a combination of game theory and BF matrix formulation in order to achieve a target spectral efficiency while keeping the interference of the secondary users towards the primary users at low levels is an interesting research approach.

Since BF aims at the minimization of undesired receptions in order to minimize interference, it can offer improved security at the physical layer. A limited number of works have proposed algorithms in this field. In [78], a topology where an untrusted AF MIMO relay that may try to decode the source's messages is studied. Two alternative solutions are developed; first, the relay is treated as an eavesdropper and does not assist the source-destination communication, while, in the second, $\mathrm{BF}$ matrices at the source and the relay are jointly designed in order to increase the secrecy rate. Moreover, in [79], a two-way relay network in the presence of an eavesdropper is studied and, through various BF schemes, the leakages are avoided and the secrecy sum rate of the two sources is increased.

Finally, regarding the formulation of precoding and $\mathrm{BF}$ matrices, other metrics, such as power consumption, should be considered. This is especially important, as relays 
may be battery-operated and the $\mathrm{BF}$ matrices they will use must achieve increased energy efficiency. The article in [80] presents a cluster of distributed relays, which form a virtual multiple-input-single-output (MISO) system. The optimal number of relays that should participate in the communication in order to satisfy an outage threshold, in terms of energy efficiency, is investigated. Results indicate that cooperative $\mathrm{BF}$ outperforms direct communication in energy and spectral efficiency.

\section{Conclusions}

In this paper, various works in the field of beamforming with multiple-input multiple-output relays have been elaborated, as they constitute an utmost promising technique for reducing interference levels and enhancing system capacity of next generation mobile broadband systems. Moreover, aiming to outline the importance of BF for MIMO relay networks while providing an overall perspective on the area, this survey includes papers that constitute the state of the art in this field. In order to facilitate the design and optimization of BF algorithms, various challenges that should be explicitly considered were presented. More specifically, important design parameters, such as the performance criteria and the power constraints, were presented and classified. Also, a literature review regarding issues such as computational complexity, and channel state information acquisition and feedback as well as antenna correlation was provided. Furthermore, the articles included in the survey were categorized based on their network topology. Firstly, articles on single-user communications were discussed and were further categorized for cases of single and multiple relaying as well as relay selection. In continuity, multiuser topologies that studied the relay broadcast channel and two-way communications were presented.

Recent research on BF MIMO relays has made significant steps, but unfortunately more research and development work is necessary towards channel impairments, synchronization, and cognitive aspects. To this end, this paper highlighted significant benefits regarding the formulation of precoding and BF matrices, interference mitigation techniques, and relay selection methods. Finally, a discussion was given on the paper's findings and on the open issues, which constitute interesting research directions in the field of $\mathrm{BF}$ with MIMO relays.

\section{Conflict of Interests}

The author declares that there is no conflict of interests regarding the publication of this paper.

\section{References}

[1] E. Telatar, "Capacity of multi-antenna Gaussian channels," European Transactions on Telecommunications, vol. 10, no. 6, pp. 585-595, 1999.

[2] A. Goldsmith, S. A. Jafar, N. Jindal, and S. Vishwanath, "Capacity limits of MIMO channels," IEEE Journal on Selected Areas in Communications, vol. 21, no. 5, pp. 684-702, 2003.
[3] D. Gesbert, M. Kountouris, R. W. Heath Jr., C.-B. Chae, and T. Sälzer, "Shifting the MIMO paradigm," IEEE Signal Processing Magazine, vol. 24, no. 5, pp. 36-46, 2007.

[4] D. Gesbert, S. Hanly, H. Huang, S. Shamai Shitz, O. Simeone, and W. Yu, "Multi-cell MIMO cooperative networks: a new look at interference," IEEE Journal on Selected Areas in Communications, vol. 28, no. 9, pp. 1380-1408, 2010.

[5] T. M. Cover and A. A. E. Gamal, "Capacity theorems for the relay channel," IEEE Transactions on Information Theory, vol. 25, no. 5, pp. 572-584, 1979.

[6] J. N. Laneman, D. N. C. Tse, and G. W. Wornell, "Cooperative diversity in wireless networks: efficient protocols and outage behavior," IEEE Transactions on Information Theory, vol. 50, no. 12, pp. 3062-3080, 2004.

[7] L. Sanguinetti, A. A. D. 'Amico, and R. Yue, "A tutorial on the optimization of amplify-and-forward MIMO relay systems," IEEE Journal on Selected Areas in Communications, vol. 30, no. 8, pp. 1331-1346, 2012.

[8] C. La Palombara, V. Tralli, B. M. Masini, and A. Conti, "Relay-assisted diversity communications," IEEE Transactions on Vehicular Technology, vol. 62, no. 1, pp. 415-421, 2013.

[9] A. Bletsas, H. Shin, and M. Z. Win, "Cooperative communications with outage-optimal opportunistic relaying," IEEE Transactions on Wireless Communications, vol. 6, no. 9, pp. 3450-3460, 2007.

[10] A. Bletsas, A. Khisti, D. P. Reed, and A. Lippman, "A simple cooperative diversity method based on network path selection," IEEE Journal on Selected Areas in Communications, vol. 24, no. 3, pp. 659-672, 2006.

[11] A. Ikhlef, D. S. Michalopoulos, and R. Schober, "Max-max relay selection for relays with buffers," IEEE Transactions on Wireless Communications, vol. 11, no. 3, pp. 1124-1135, 2012.

[12] N. Nomikos, D. Vouyioukas, T. Charalambous, I. Krikidis, D. N. Skoutas, and M. Johansson, "Capacity improvement through buffer-aided successive opportunistic relaying," in Proceedings of the IEEE Wireless Vitae Conference, June 2013.

[13] N. Nomikos, T. Charalambous, I. Krikidis, D. N. Skoutas, D. Vouyioukas, and M. Johansson, "Buffer-aided successive opportunistic relaying with inter-relay interference cancellation," in Proceedings of the IEEE International Symposium on Personal Indoor and Mobile Radio Communication, September 2013.

[14] P. Balaban and J. Salz, "Dual diversity combining and equalization in digital cellular mobile radio," IEEE Transactions on Vehicular Technology, vol. 40, no. 2, pp. 342-354, 1991.

[15] F. Rashid-Farrokhi, K. J. R. Liu, and L. Tassiulas, "Transmit beamforming and power control for cellular wireless systems," IEEE Journal on Selected Areas in Communications, vol. 16, no. 8, pp. 1437-1450, 1998.

[16] S. Bellofiore, C. A. Balanis, J. Foutz, and A. S. Spanias, "Smartantenna systems for mobile communication networks. Part 1 : overview and antenna design," IEEE Antennas and Propagation Magazine, vol. 44, no. 3, pp. 145-154, 2002.

[17] S. Bellofiore, J. Foutz, C. A. Balanis, and A. S. Spanias, "Smartantenna system for mobile communication networks Part 2: beamforming and network throughput," IEEE Antennas and Propagation Magazine, vol. 44, no. 4, pp. 106-114, 2002.

[18] S. Berger, M. Kuhn, A. Wittneben, T. Unger, and A. Klein, "Recent advances in amplify-and-forward two-hop relaying," IEEE Communications Magazine, vol. 47, no. 7, pp. 50-56, 2009.

[19] Y. Hua, "An overview of beamforming and power allocation for MIMO relays," in Proceedings of the IEEE Military Communications Conference, pp. 375-380, October 2010. 
[20] D. P. Palomar, J. M. Cioffi, and M. A. Lagunas, "Joint TxRx beamforming design for multicarrier MIMO channels: a unified framework for convex optimization," IEEE Transactions on Signal Processing, vol. 51, no. 9, pp. 2381-2401, 2003.

[21] A. S. Behbahani, R. Merched, and A. M. Eltawil, "Optimizations of a MIMO relay network," IEEE Transactions on Signal Processing, vol. 56, no. 10, pp. 5062-5073, 2008.

[22] P. Wu and R. Schober, "Cooperative beamforming for singlecarrier frequency-domain equalization systems with multiple relays," IEEE Transactions on Wireless Communications, vol. 11, no. 6, pp. 2276-2286, 2012.

[23] Y. Rong and F. Gao, "Optimal beamforming for non-regenerative MIMO relays with direct link," IEEE Communications Letters, vol. 13, no. 12, pp. 926-928, 2009.

[24] F.-S. Tseng, M.-Y. Chang, and W.-R. Wu, "Joint MMSE transceiver design in amplify-and-forward mimo relay systems with tomlinson-harashima source precoding," in Proceedings of the IEEE 21st International Symposium on Personal Indoor and Mobile Radio Communications, pp. 443-448, September 2010.

[25] Y. Rong, "Optimal joint source and relay beamforming for MIMO relays with direct link," IEEE Communications Letters, vol. 14, no. 5, pp. 390-392, 2010.

[26] C. Xing, S. Ma, M. Xia, and Y. C. Wu, "Cooperative beamforming for dual-hop amplify-and-forward multi-antenna relaying cellular networks," Elsevier Signal Processing, vol. 92, no. 11, pp. 2689-2699, 2012.

[27] R. Wang, M. Tao, and Y. Huang, "Linear precoding designs for amplify-and-forward multiuser two-way relay systems," IEEE Transactions on Wireless Communications, vol. 11, no. 12, pp. 4457-4469, 2012.

[28] J. Joung and A. H. Sayed, "Multiuser two-way amplify-andforward relay processing and power control methods for beamforming systems," IEEE Transactions on Signal Processing, vol. 58, no. 3, pp. 1833-1846, 2010.

[29] Y. Rong, "Joint source and relay optimization for two-way MIMO multi-relay networks," IEEE Communications Letters, vol. 15, no. 12, pp. 1329-1331, 2011.

[30] C. Chen, L. Bai, B. Wu, and J. Choi, "Relay selection and beamforming for cooperative bi-directional transmissions with physical layer network coding," IET Communications, vol. 5, no. 14, pp. 2059-2067, 2011.

[31] Z. B. Wang, L. J. Xiang, L. H. Zheng, and H. Ding, "MSMSEbased optimal beamforming design and simplification on AF MIMO two-way relay channels," Springer Journal of Central Southern University, vol. 19, pp. 465-470, 2012.

[32] Z. Hui, Y. Longxiang, and Z. Hongbo, "Precoding and decoding design for two-way MIMO AF multiple-relay system," Journal of Electronics, vol. 29, no. 3, pp. 177-189, 2012.

[33] Y.-W. Liang and R. Schober, "Cooperative amplify-and-forward beamforming with multi-antenna source and relays," in Proceedings of the 3rd IEEE International Workshop on Computational Advances in Multi-Sensor Adaptive Processing, pp. 277280, December 2009.

[34] B. Khoshnevis, W. Yu, and R. Adve, "Grassmannian beamforming for MIMO amplify-and-forward relaying," IEEE Journal on Selected Areas in Communications, vol. 26, no. 8, pp. 1397-1407, 2008.

[35] Y. Zhao, R. Adve, and T. J. Lim, "Beamforming with limited feedback in amplify-and-forward cooperative networks[transactions letters]," IEEE Transactions on Wireless Communications, vol. 7, no. 12, pp. 5145-5149, 2008.
[36] B. K. Chalise, L. Vandendorpe, Y. D. Zhang, and M. G. Amin, "Local CSI based selection beamforming for amplify-andforward MIMO relay networks," IEEE Transactions on Signal Processing, vol. 60, no. 5, pp. 2433-2446, 2012.

[37] R. H. Y. Louie, Y. Li, H. A. Suraweera, and B. Vucetic, "Performance analysis of beamforming in two hop amplify and forward relay networks with antenna correlation," IEEE Transactions on Wireless Communications, vol. 8, no. 6, pp. 3132-3141, 2009.

[38] Z. Zhou and B. Vucetic, "An optimized cooperative beamforming scheme in MIMO relay broadcast channels," in Proceedings of the IEEE Global Telecommunications Conference, pp. 1-6, December 2009.

[39] Z. Zhou and B. Vucetic, "A cooperative beamforming scheme in mimo relay broadcast channels," IEEE Transactions on Wireless Communications, vol. 10, no. 3, pp. 940-947, 2011.

[40] B. Zhang, Z. He, K. Niu, and L. Zhang, "Robust linear beamforming for MIMO relay broadcast channel with limited feedback," IEEE Signal Processing Letters, vol. 17, no. 2, pp. 209212, 2010

[41] E. Yilmaz, R. Zakhour, D. Gesbert, and R. Knopp, "Multi-pair two-way relay channel with multiple antenna relay station," in Proceedings of the IEEE International Conference on Communications, pp. 1-5, May 2010.

[42] A. El-Keyi and B. Champagne, "Adaptive linearly constrained minimum variance beamforming for multiuser cooperative relaying using the kalman filter," IEEE Transactions on Wireless Communications, vol. 9, no. 2, pp. 641-651, 2010.

[43] Y. Liu and A. P. Petropulu, "Cooperative beamforming in multisource multi-destination relay systems with SINR constraints," in Proceedings of the IEEE International Conference on Acoustics, Speech, and Signal Processing, pp. 2870-2873, March 2010.

[44] Y. Fan and J. Thompson, "MIMO configurations for relay channels: theory and practice," IEEE Transactions on Wireless Communications, vol. 6, no. 5, pp. 1774-1786, 2007.

[45] X. Tang and Y. Hua, "Optimal design of non-regenerative MIMO wireless relays," IEEE Transactions on Wireless Communications, vol. 6, no. 4, pp. 1398-1407, 2007.

[46] E. Baccarelli, M. Biagi, C. Pelizzoni, and N. Cordeschi, "Maximum-rate node selection for power-limited multiantenna relay backbones," IEEE Transactions on Mobile Computing, vol. 8, no. 6, pp. 807-820, 2009.

[47] M. A. Torabi and J.-F. Frigon, "Semi-orthogonal relay selection and beamforming for amplify-and-forward MIMO relay channels," in Proceedings of the IEEE Wireless Communications and Networking Conference, pp. 48-53, April 2008.

[48] G. Okeke, W. A. Krzymien, and Y. Jing, "Beamforming in nonregenerative MIMO broadcast relay networks," IEEE Transactions on Signal Processing, vol. 60, no. 12, pp. 6641-6654, 2012.

[49] H. Wan, W. Chen, and X. Wang, "Joint source and relay design for MIMO relaying broadcast channels," IEEE Communications Letters, vol. 17, no. 2, pp. 345-348, 2013.

[50] K. T. Truong and R. W. Heath, "Joint transmit precoding for the relay interference broadcast channel," IEEE Transactions on Vehicular Technology, vol. 62, no. 3, pp. 1201-1215, 2013.

[51] K. Lee, S.-H. Park, J.-S. Kim, and I. Lee, "Degrees of freedom on mimo multi-link two-way relay channels," in Proceedings of the 53rd IEEE Global Communications Conference, pp. 1-5, December 2010.

[52] Z. Ding, I. Krikidis, J. Thompson, and K. K. Leung, "Physical layer network coding and precoding for the two-way relay channel in cellular systems," IEEE Transactions on Signal Processing, vol. 59, no. 2, pp. 696-712, 2011. 
[53] N. Lee, C.-B. Chae, O. Simeone, and J. Kang, "On the optimization of two-way AF MIMO relay channel with beamforming," in Proceedings of the 44th Asilomar Conference on Signals, Systems and Computers, pp. 918-922, November 2010.

[54] R. Zhang, Y.-C. Liang, C. C. Chai, and S. Cui, "Optimal beamforming for two-way multi-antenna relay channel with analogue network coding," IEEE Journal on Selected Areas in Communications, vol. 27, no. 5, pp. 699-712, 2009.

[55] S. Mohajer, S. N. Diggavi, and D. N. C. Tse, "Approximate capacity of a class of gaussian relay-interference networks," in Proceedings of the IEEE International Symposium on Information Theory, pp. 31-35, July 2009.

[56] Y. Shi, J. Zhang, and K. B. Letaief, "Coordinated relay beamforming for amplify-and-forward two-hop interference networks," in Proceedings of the IEEE Global Communications Conference, pp. 2408-2413, December 2012.

[57] K. T. Truong and R. W. Heath Jr., "Relay beamforming using interference pricing for the two-hop interference channel," in Proceedings of the 54th Annual IEEE Global Telecommunications Conference, pp. 1-5, December 2011.

[58] T. M. Kim, B. Bandemer, and A. Paulraj, "Beamforming for network-coded MIMO two-way relaying," in Proceedings of the 44th Asilomar Conference on Signals, Systems and Computers, pp. 647-652, November 2010.

[59] G. Ye and R. Wang, "Transceiver designs for multiuser two-way relay system," in Proceedings of the International Workshop on Information and Electronics Engineering, pp. 4186-4191, March 2012.

[60] S. Borade, L. Zheng, and R. Gallager, "Amplify-and-forward in wireless relay networks: rate, diversity, and network size," IEEE Transactions on Information Theory, vol. 53, no. 10, pp. 33023318, 2007.

[61] Y.-W. Liang and R. Schober, "Cooperative amplify-and-forward beamforming with multiple multi-antenna relays," IEEE Transactions on Communications, vol. 59, no. 9, pp. 2605-2615, 2011.

[62] Z. Bai, Y. Xu, D. Yuan, and K. Kwak, "Performance analysis of cooperative MIMO system with relay selection and power allocation," in Proceedings of the IEEE International Conference on Communications, pp. 1-5, May 2010.

[63] C.-K. Wen, K.-K. Wong, and J.-C. Chen, "Precoding design in MIMO multiple-access cellular relay systems with partial CSI," in Proceedings of the IEEE Wireless Communications and Networking Conference, pp. 1-6, April 2010.

[64] F. Gao, T. Cuiy, B. Jiangz, and X. Gaoz, "On communication protocol and beamforming design for amplify-and-forward NWay relay networks," in Proceedings of the 3rd IEEE International Workshop on Computational Advances in Multi-Sensor Adaptive Processing, pp. 109-112, December 2009.

[65] B. Xie, D. Munoz, and H. Minn, "A reduced overhead OFDM relay system with clusterwise TMRC beamforming," IEEE Communications Letters, vol. 16, no. 12, pp. 1913-1916, 2012.

[66] S. W. Peters and R. W. Heath, "Nonregenerative MIMO relaying with optimal transmit antenna selection," IEEE Signal Processing Letters, vol. 15, pp. 421-424, 2008.

[67] H.-N. Cho, J.-W. Lee, A.-Y. Kim, and Y.-H. Lee, "Cooperative interference mitigation with partial CSI in multi-user dual-hop MISO relay channels," in Proceedings of the 20th IEEE Personal, Indoor and Mobile Radio Communications Symposium, pp. 12111215, September 2009.

[68] H. A. Suraweera, H. K. Garg, and A. Nallanathan, "Beamforming in dual-hop fixed gain relay systems with antenna correlation," in Proceedings of the IEEE International Conference on Communications, pp. 1-5, May 2010.

[69] N. S. Ferdinand and N. Rajatheva, "Unified performance analysis of two-hop amplify-and-forward relay systems with antenna correlation," IEEE Transactions on Wireless Communications, vol. 10, no. 9, pp. 3002-3011, 2011.

[70] T. Riihonen, A. Balakrishnan, K. Haneda, S. Wyne, S. Werner, and R. Wichman, "Optimal eigenbeamforming for suppressing self-interference in full-duplex MIMO relays," in Proceedings of the 45th Annual Conference on Information Sciences and Systems, pp. 1-6, March 2011.

[71] S. M. Kim and M. Bengtsson, "Virtual full-duplex buffer-aided relaying-relay selection and beamforming," in Proceedings of the IEEE International Symposium on Personal Indoor and Mobile Radio Communication, September 2013.

[72] M. K. Maggs, S. G. O’Keefe, and D. V. Thiel, "Consensus clock synchronization for wireless sensor networks," IEEE Sensors Journal, vol. 12, no. 6, pp. 2269-2277, 2012.

[73] A. G. Kanatas, A. Kalis, and G. P. Efthymoglou, "A single hop architecture exploiting cooperative beamforming for wireless sensor networks," Physical Communication, vol. 4, no. 3, pp. 237-243, 2011.

[74] N. Nomikos, P. Makris, D. Vouyioukas, D. N. Skoutas, and C. Skianis, "Distributed joint relay-pair selection for buffer-aided successive opportunistic relaying," in Proceedings of the IEEE International Workshop on Computer-Aided Modeling Analysis of Design of Communication Links and Networks, September 2013.

[75] L. Chen, K.-K. Wong, H. Chen, J. Liu, and G. Zheng, "Optimizing transmitter-receiver collaborative-relay beamforming with perfect CSI," IEEE Communications Letters, vol. 15, no. 3, pp. 314-316, 2011.

[76] T. Luan, F. Gao, X. D. Zhang, J. C. F. Li, and M. Lei, "Rate maximization and beamforming design for relay-aided multiuser cognitive networks," IEEE Transactions on Vehicular Technology, vol. 61, no. 4, pp. 1940-1945, 2012.

[77] Q. Li, Q. Zhang, R. Feng, L. Luo, and J. Qin, "Optimal relay selection and beamforming in MIMO cognitive multi-relay networks," IEEE Communications Letters, vol. 17, no. 6, pp. 11881191, 2013.

[78] C. Jeong, I.-M. Kim, and D. I. Kim, "Joint secure beamforming design at the source and the relay for an amplify-and-forward MIMO untrusted relay system," IEEE Transactions on Signal Processing, vol. 60, no. 1, pp. 310-325, 2012.

[79] H. M. Wang, Y. Qinye, and X. G. Xia, "Distributed beamforming for physical-layer security of two-way relay networks," IEEE Transactions on Signal Processing, vol. 60, no. 7, pp. 3532-3545, 2012.

[80] G. Lim and L. J. Cimini, "Energy-efficient cooperative beamforming in clustered wireless networks," IEEE Transactions on Wireless Communications, vol. 12, no. 3, pp. 1376-1385, 2013. 

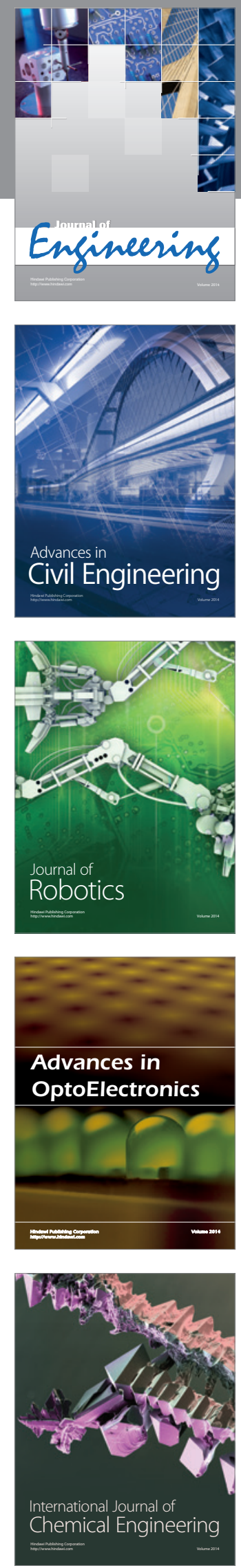

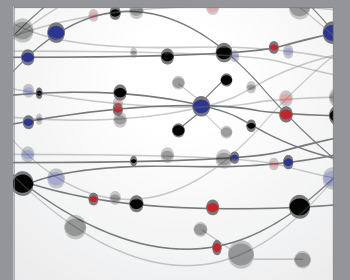

The Scientific World Journal
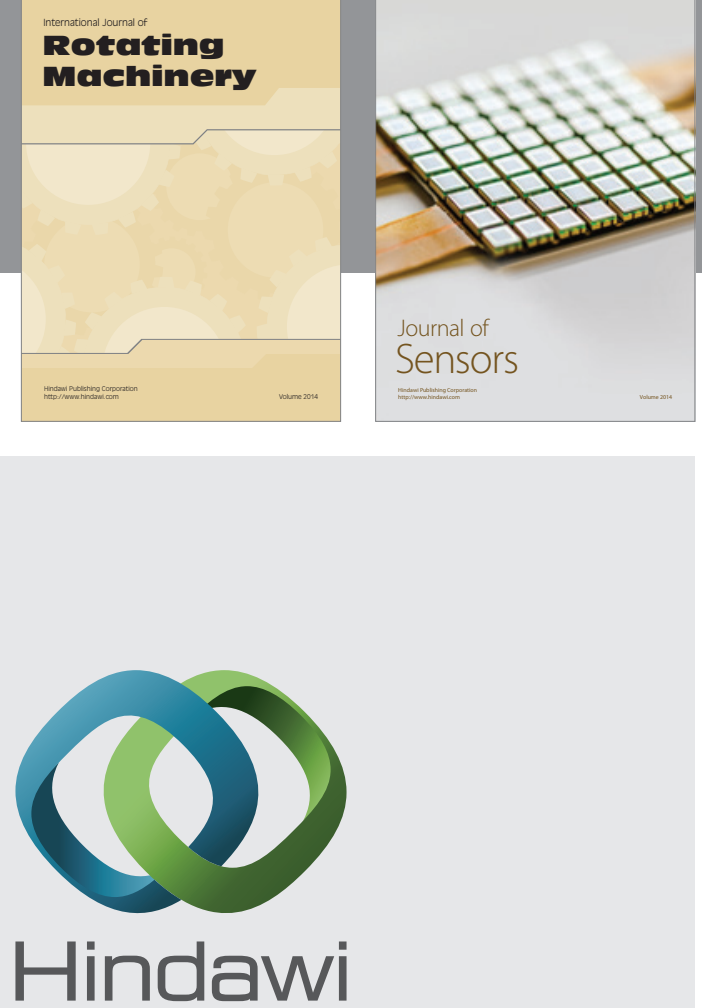

Submit your manuscripts at http://www.hindawi.com
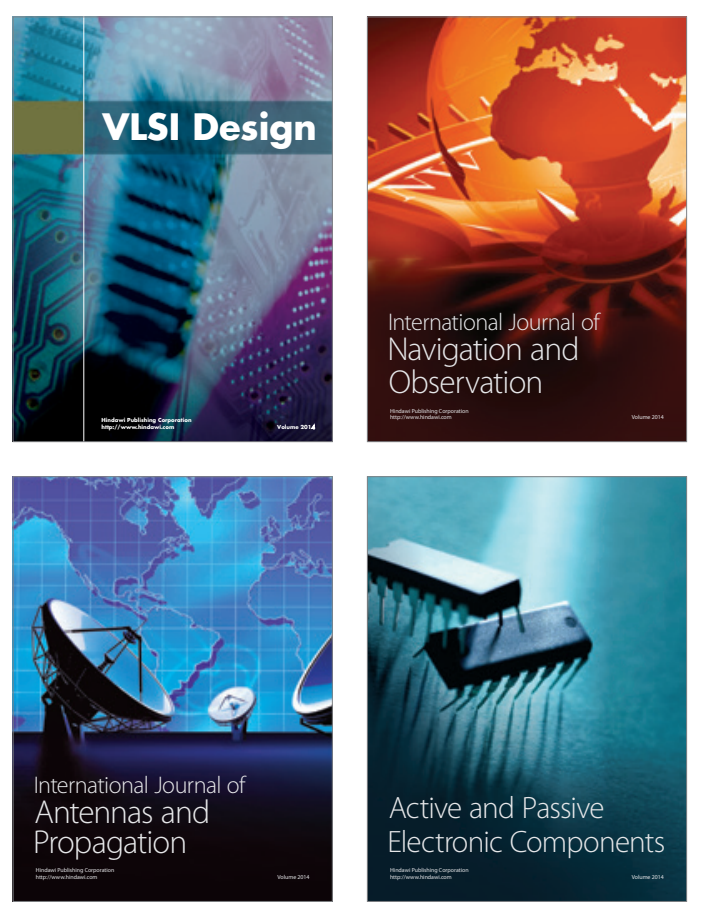
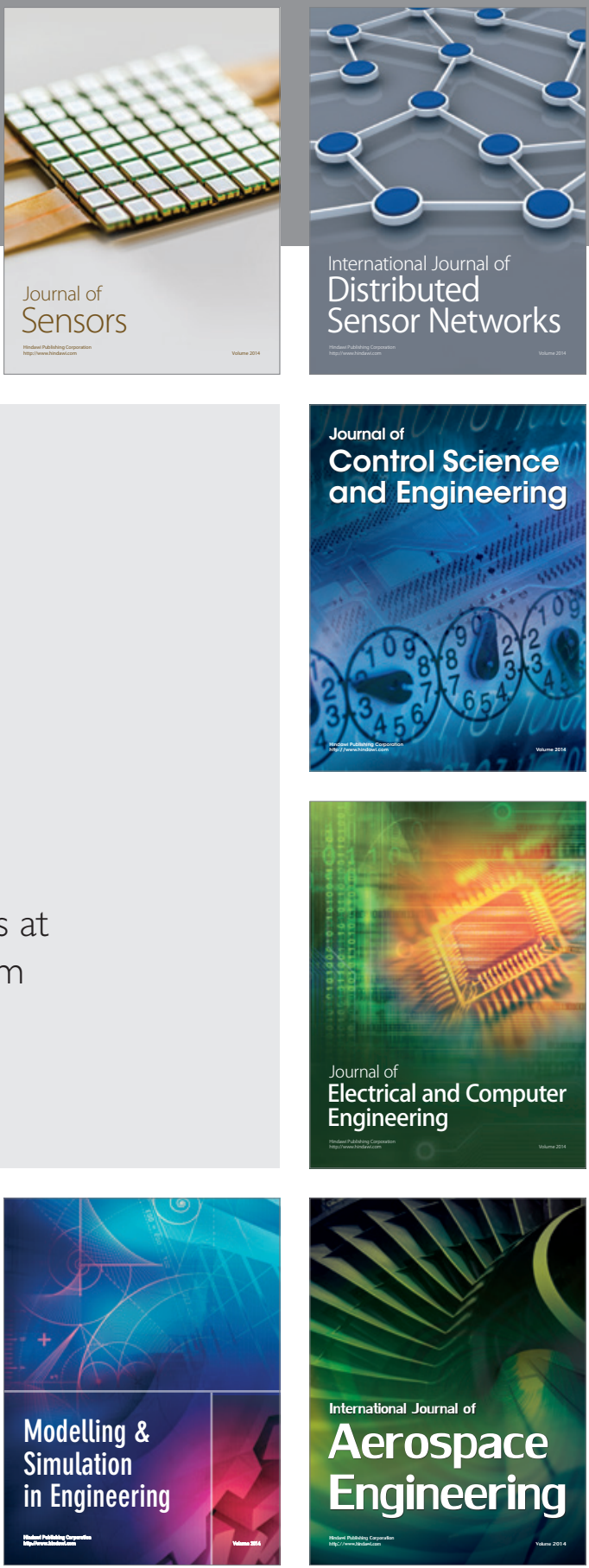

Journal of

Control Science

and Engineering
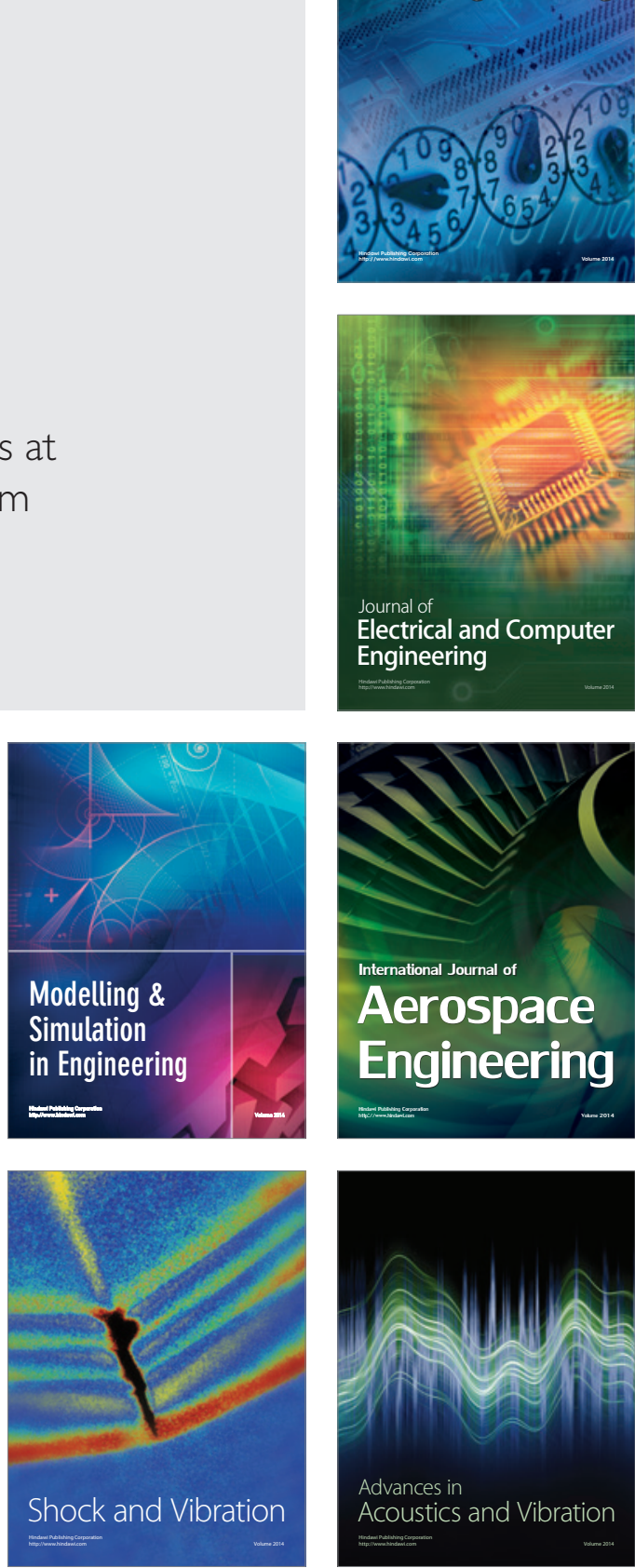